ADP-01-35/T467

\title{
Structure Functions of Unstable Lithium Isotopes
}

\author{
K. Saito ${ }^{a *}$, M. Ueda ${ }^{b \dagger}$, K. Tsushima ${ }^{c \ddagger}$ and A.W. Thomas ${ }^{c \S}$ \\ a Tohoku College of Pharmacy, Sendai 981-8558, Japan \\ ${ }^{b}$ Institute of Physics, University of Tsukuba, Tsukuba 305-8571, Japan \\ ${ }^{c}$ Department of Physics and Mathematical Physics and \\ Special Research Center for the Subatomic Structure of Matter (CSSM) \\ Adelaide University, Adelaide SA 5005, Australia
}

\begin{abstract}
We study both the spin-average and spin-dependent structure functions of the lithium isotopes, ${ }^{6-11} \mathrm{Li}$, which could be measured at RIKEN and other nuclear facilities in the future. It is found that the light-cone momentum distribution of the valence neutron in the halo of ${ }^{11} \mathrm{Li}$ is very sharp and symmetric around $y=1$, because of the weak binding. This implies that such neutrons are in an environment very close to that of a free neutron. The EMC ratios for Li isotopes are then calculated. Furthermore we investigate a new ratio, $R_{d}$, of the difference between the Li structure functions of mass number $A\left(F_{2}^{A}\right)$ and $A-1\left(F_{2}^{A-1}\right)$ to the difference between the structure functions of the deuteron and free proton. We study the possibility of extracting the neutron structure function from data for the nuclear structure functions of the $\mathrm{Li}$ isotopes. In particular, the ratios $R_{n}$ for $A=9$ and 11 present an attractive possibility for extracting the free neutron structure function. Next we calculate the spin-dependent structure functions of ${ }^{7,9,11} \mathrm{Li}$ isotopes, which have spin of $3 / 2$. For such nuclei the spin structure function is given in terms of the multipole spin structure functions; ${ }_{1}^{3 / 2} g_{1}$, which is analogous to the usual spin structure function, $g_{1}$, for a target with spin $1 / 2$, and a new one, ${ }_{3}^{3 / 2} g_{1}$, which first arises for a target with spin $3 / 2$. The effect of the nuclear binding and Fermi motion on ${ }_{1}^{3 / 2} g_{1}$ is about $10 \%$ in the region $x<0.7$, but it becomes quite important at large $x$. The spin structure function of ${ }_{3}^{3 / 2} g_{1}$
\end{abstract}

\footnotetext{
*E-mail address: ksaito@nucl.phys.tohoku.ac.jp

$\dagger^{\dagger}$ mueda@nucl.ph.tsukuba.ac.jp

${ }^{\ddagger}$ ktsushim@physics.adelaide.edu.au. Address from November 1, 2001; Department of Physics and Astronomy, University of Georgia, Athens, GA 30602, USA (tsushima@hal.physast.uga.edu)

§athomas@physics.adelaide.edu.au
} 
is negative at small $x$ but it becomes positive in the region $0.2<x<0.5$. However, the magnitude is very small. At large $x$ it is again negative and its absolute value becomes large because of the Fermi motion. Finally, we discuss the modification of the Gottfried and Bjorken integrals in a nuclear medium and point out several candidates for a pair of mirror nuclei to study the flavor-nonsinglet quark distributions in nuclei.

PACS: 25.30.Mr; 13.60.Hb; 24.85.+p

Keywords: Nuclear structure functions; Neutron structure functions; Unstable nuclei 


\section{INTRODUCTION}

The recent development of secondary radioactive beams with sufficient intensities, which are produced through high energy fragmentation of nuclei, has opened a new frontier in nuclear physics, i.e., the study of nuclei with extreme ratios of the numbers of neutrons, $N$, to the number of protons, $Z$. Such nuclei, far from the stability line, have been investigated intensively at many nuclear facilities in the world as one of the main subjects of nuclear science today [1]. There are various of interesting problems such as weakly bound nuclei, lowdensity nuclear matter, novel nuclear structures of halo and skin, very asymmetric nuclear matter, appearance/disappearance of magic numbers in the vicinity of the neutron and proton drip line [1,2].

In particular, it is noticeable that the halo structure in some light nuclei was discovered on and close to the neutron drip line [3]. Those nuclei have spatial extensions much larger than naive expectations based on their mass numbers. A typical example is ${ }^{11} \mathrm{Li}$, which has a root mean square $(\mathrm{rms})$ radius of about $3.1 \mathrm{fm}$. In Table I $\mathrm{f}$ we summarize the observed data of ${ }^{6-11} \mathrm{Li}$ isotopes [4,5]. The experimental data suggests that nuclei like ${ }^{11} \mathrm{Li}$ could be understood as a core surrounded by one or two more neutrons, which extend to several times the nuclear radius - the so-called halo nuclei. This picture is also supported by recent experiments with break-up of loosely bound neutron-rich projectiles on light targets [6].

The $\beta$-unstable nuclei in the vicinity of the neutron drip line have two distinctive features. The first feature is a large asymmetry in $Z$ and $N$. For stable nuclei with $Z \geq 3$, due to the Coulomb interaction and the nuclear saturation property, (i) the ratio $N / Z$ is $1.0 \sim 1.6$, (ii) the observed central density is $\sim 0.17 \mathrm{fm}^{-3}$ except for very light nuclei, and (iii) the density distributions of protons and neutrons are similar and the thickness of the nuclear surface is almost unchanged for all nuclei. For $\beta$-unstable nuclei close to the neutron drip line, the $N / Z$ ratio is about $2 \sim 4$ and the distributions of protons and neutrons are considerably decoupled (i.e., neutron halo and neutron skin).

The second feature is a small separation energy of the valence neutron. For light stable nuclei, where $N \approx Z$, the separation energy of the last nucleon is usually several $\mathrm{MeV}$. On the other hand, with an increasing number of neutrons the separation energy of the last neutron, $S_{n}$, varies from several $\mathrm{MeV}$ to less than $1 \mathrm{MeV}$ at the neutron drip line, while the separation energy of a proton tends to increase and sometimes exceeds $10 \mathrm{MeV}$ near the neutron drip line. This difference between the separation energy of a neutron and that of a proton implies a difference in the Fermi energies of neutrons and protons, and it provides an understanding of the mechanism of formation of neutron halos and skins as well as the instability of $\beta$-unstable nuclei.

The partonic distribution functions in such nuclei are of considerable interest in view of these features [7]. The distributions of quarks in a nucleus differ significantly from those in the free nucleon - the so-called European Muon Collaboration (EMC) effect [8,9]. Since the early 80's a large amount of data on flavor-singlet nuclear structure functions, i.e., $N \simeq Z$, has been collected. However, relatively less attention has been paid to the flavornonsinglet structure functions of a nucleus. Measurements of nonsinglet nuclear structure functions in deep inelastic scattering (DIS) could shed light on many phenomena involving nonperturbative QCD in nuclei, such as SU(3) symmetry breaking, flavor asymmetry [10] and so on. 
Recently we have studied the nonsinglet structure function of the lightest mirror nuclei $\left({ }^{3} \mathrm{He}\right.$ and $\left.{ }^{3} \mathrm{H}\right)$ [7, 11 and pointed out that the nonsinglet structure function in those nuclei is enhanced at small $x$ by nuclear shadowing. Furthermore, we suggested that the Gottfried integral is generally divergent because of charge symmetry breaking. We have also investigated the effect of the medium modification of pion fields on the flavor-nonsinglet structure function of the lightest mirror nuclei [12]. It is intriguing to study such nonsinglet structure functions using a pair of unstable nuclei [7] because such nuclei could give rise to a large asymmetry in isospin space - recall the first feature for unstable nuclei discussed above. The current rapid development of radioactive nuclear beam techniques at many nuclear facilities in the world will provide good opportunities for advancement to the study of nonsinglet structure functions in the near future [2, 13].

Utilizing the second feature of neutron-rich nuclei far from the stability line, i.e., a wide range of the neutron separation energy, we could investigate the binding (off-mass shell [14 and shell structure dependences) and Fermi motion effects on nuclear structure functions [7]. Furthermore, the neutron structure function, $F_{2}^{n}$, could be extracted from nuclear structure functions of neutron-rich nuclei, by virtue of the weakness of the neutron separation energy [7].

It is very important to determine the neutron structure function from nuclear experimental data. The proton structure function, $F_{2}^{p}$, which at intermediate and large- $x$ measures a charge-squared weighted combination of the valence $u$ and $d$ distributions, depends mostly on the $u$ quark distributions owing to the larger weight of the $u$ quark charge. It is therefore necessary to know the neutron structure function as precisely as possible to determine the individual isospin distributions separately.

The problem of extracting neutron structure functions from nuclear data is rather old [15]. Because of the absence of a free neutron target the deuteron is traditionally used as an effective neutron target: $F_{2}^{n} \approx F_{2}^{d}-F_{2}^{p}$ ( $F_{2}^{d}$ being the deuteron structure function). While this approximation may be valid for $0.1<x<0.4$, it breaks down dramatically for large $x$ because of the Fermi motion. The Adelaide group [16] has recently reanalyzed the deuteron structure function to determine $F_{2}^{n}$, and they showed that the nuclear binding and Fermi motion effects were significantly larger than those in earlier analyses. In particular, ignoring the effect of nuclear binding yields an error of up to $50 \%$ in the ratio $F_{2}^{n} / F_{2}^{p}$ extracted at $x \sim 0.75$.

Recently, the $A=3$ system $\left({ }^{3} \mathrm{He}\right.$ and $\left.{ }^{3} \mathrm{H}\right)$ is also being seriously considered as an alternative way to extract the neutron structure function [17,18]. Using realistic Faddeev wave functions, it was shown that $F_{2}^{n}$ could be extracted from the ratio of those structure functions, where the nuclear effects are cancel each other so that the ratio is within $2 \%$ of unity for $x<0.85$. In the present paper we discuss another way to extract the neutron structure function using the radioactive $\mathrm{Li}$ isotopes. This is based on the novel idea that the valence neutrons in the $\beta$-unstable nuclei are so loosely bound that they may be regarded as essentially as free neutrons.

For the last decade polarized DIS experiments have yielded a number of important results concerning the spin structure of the nucleon. The measurements of the proton spin structure function, $g_{1}^{p}$, by EMC [19], combined with flavor-nonsinglet matrix elements from weak decays, gave significant information on the singlet axial charge of the proton. The small size of this resulted in the so-called proton spin crisis, and it has been reanalyzed 
by many groups [20]. As an extra source of information, it is important to measure the neutron spin structure function, $g_{1}^{n}$, for testing the fundamental Bjorken sum rule of the free nucleon. However, as in the unpolarized case, the absence of the free neutron target means that nuclei have to be used for this purpose. In fact, to study $g_{1}^{n}$ the SLAC E142 Collaboration [21] has measured the spin structure function of ${ }^{3} \mathrm{He}$, while the Spin Muon Collaboration (SMC) [22] has observed the $g_{1}$ structure function of the deuteron. (For recent experiments, see Ref. [23].)

The nuclear binding, Fermi motion and shadowing modify the parton distributions in a nuclear medium. As in the case of Gottfried sum rule in nuclei [7, [1], it is worthwhile to study how the flavor-nonsinglet combination of the spin structure functions, which appears in the Bjorken sum rule, is modified in a nucleus. We can discuss it again using a pair of polarized mirror nuclei - the promising pairs may be $\left({ }^{3} \mathrm{He},{ }^{3} \mathrm{H}\right)$ [24] and $\left({ }^{7} \mathrm{Li},{ }^{7} \mathrm{Be}\right.$ ) [25] (we will discuss several candidates in Sec. V). If the spin structure functions of unstable nuclei were measured with a certain accuracy, one could obtain significant information on the spin structure of partons in nuclei.

In general, the spin-average DIS cross section, $\bar{\sigma}$, and polarization asymmetry, $\Delta \sigma$, of a target nucleus with mass $M_{T}$ and spin $J$ in the scaling limit is given by 26]

$$
\begin{aligned}
& \frac{d \bar{\sigma}^{J}}{d x d y}=C x\left[1+(1-y)^{2}\right] \sum_{L=\text { even }}{ }_{L}^{J} F_{1}(x) \rho_{L}^{J}, \\
& \frac{d \Delta \sigma^{J}}{d x d y}=C x\left[1-(1-y)^{2}\right] \sum_{L=\text { odd }}{ }_{L}^{J} g_{1}(x) \rho_{L}^{J},
\end{aligned}
$$

with a kinematical factor $C=e^{4} M_{T} E / 2 \pi Q^{4}$, where $E$ is the incident lepton energy, $q^{2}(=$ $\left.-Q^{2}\right)$ the four momentum transfer squared and $y$ the ratio of the energy transfer to $E$ in the target rest frame. ${ }_{L}^{J} F_{1}$ and ${ }_{L}^{J} g_{1}$ are, respectively, multipole spin-average and multipole spindependent structure functions, which are defined in terms of the target helicity-dependent structure functions, $F_{1}^{J H}$ and $g_{1}^{J H}$ ( $H$ stands for helicity), and a projection matrix, $M_{L}^{J}$ :

$$
{ }_{L}^{J} F_{1}=\operatorname{tr}\left[M_{L}^{J} F_{1}^{J}\right], \quad{ }_{L}^{J} g_{1}=\operatorname{tr}\left[M_{L}^{J} g_{1}^{J}\right] .
$$

Here $F_{1}^{J}$ and $g_{1}^{J}$ are $(2 J+1) \times(2 J+1)$ diagonal matrices with their elements $\left(F_{1}^{J}\right)_{H H}=F_{1}^{J H}$ and $\left(g_{1}^{J}\right)_{H H}=g_{1}^{J H}$, respectively, and $M_{L}^{J}$ is defined as

$$
\left(M_{L}^{J}\right)_{H H^{\prime}}=(-)^{J-L}(J H, J-H \mid L 0) \delta_{H H^{\prime}},
$$

where $(J H, J-H \mid L 0)$ is the Clebsch-Gordan coefficient. In Eqs. (1) and (2) $\rho_{L}^{J}$ is a multipole projection of a spin density matrix, $\rho^{J}$. The multipole structure functions are irreducible under rotation. (Note that in the scaling limit ${ }_{L}^{J} F_{2}$ is related to ${ }_{L}^{J} F_{1}$ through the Callan-Gross relation.)

For a target with spin $J=1$ the DIS cross section is given in terms of three non-vanishing multipole structure functions, ${ }_{0}^{1} F_{1},{ }_{1}^{1} g_{1}$ and ${ }_{2}^{1} F_{1}$, in the scaling limit. This case corresponds, for example, to the DIS on ${ }^{6} \mathrm{Li}$ (see Table $\mathbb{1}$ ). The first one is then related to the usual spin-average structure functions, $F_{1}$, through

$$
{ }_{0}^{J} F_{1}(x)=\frac{1}{\sqrt{2 J+1}} \sum_{H}\left[q_{\uparrow}^{J H}(x)+q_{\downarrow}^{J H}(x)\right]=2 \sqrt{2 J+1} F_{1}(x),
$$


where $q_{s}^{J H}$ is the distribution function of quarks with spin $s$ in the target. (Note that all flavor labels and charges are suppressed.) The usual $F_{2}$ structure function can be obtained using the Callan-Gross relation. The second one corresponds to the spin structure function for a spin- $1 / 2$ target, $g_{1}$, and they are related as ${ }_{1}^{1} g_{1}=\sqrt{2} g_{1}$. The last one is the quadrupole structure function for a spin-1 target, which is sometimes called $b_{1}$ [27].

The ${ }^{8} \mathrm{Li}$ target has spin-2 (see Table $\mathbb{\text { I) }}$. In such a case there are five non-trivial structure functions in the scaling limit: ${ }_{0}^{2} F_{1},{ }_{1}^{2} g_{1},{ }_{2}^{2} F_{1},{ }_{3}^{2} g_{1}$ and ${ }_{4}^{2} F_{1}$. Then, ${ }_{0}^{2} F_{1},{ }_{1}^{2} g_{1}$ and ${ }_{2}^{2} F_{1}$ are analogous to $F_{1}, g_{1}$ and $b_{1}$, respectively, for $J=1$. The structure function ${ }_{3}^{2} g_{1}$ is analogous to the one that first appears at $J=3 / 2,{ }_{3}^{3 / 2} g_{1}$ (see below). The last one, ${ }_{4}^{2} F_{1}$, first arises for $J=2$.

The spin of Li isotopes with odd $A$ is $J=3 / 2$ (see Table $\mathbb{\text { I) }}$ ). In this case there are four non-vanishing multipole structure functions in the scaling limit: ${ }_{0}^{3 / 2} F_{1},{ }_{2}^{3 / 2} F_{1},{ }_{1}^{3 / 2} g_{1}$ and ${ }_{3}^{3 / 2} g_{1}$. The first one is again proportional to the usual spin-average structure functions, and the second one is analogous to the quadrupole structure function, $b_{1}$, for a spin- 1 target. The last two are sensitive to quark spin asymmetries. ${ }_{1}^{3 / 2} g_{1}$ is the analog to $g_{1}$ for a spin- $1 / 2$ target:

$$
{ }_{1}^{3 / 2} g_{1}(x)=\frac{1}{\sqrt{20}}\left[3 q_{\uparrow}^{3 / 23 / 2}(x)-3 q_{\downarrow}^{3 / 23 / 2}(x)+q_{\uparrow}^{3 / 21 / 2}(x)-q_{\downarrow}^{3 / 21 / 2}(x)\right]
$$

while ${ }_{3}^{3 / 2} g_{1}$ is a new asymmetry:

$$
{ }_{3}^{3 / 2} g_{1}(x)=\frac{1}{\sqrt{20}}\left[q_{\uparrow}^{3 / 23 / 2}(x)-q_{\downarrow}^{3 / 23 / 2}(x)-3 q_{\uparrow}^{3 / 21 / 2}(x)+3 q_{\downarrow}^{3 / 21 / 2}(x)\right] .
$$

It is important to keep in mind that all nucleons contribute to the spin-average structure functions but that only unpaired valence nucleons mainly contribute to the other multipole structure functions.

In the present paper we concentrate on the spin-average structure functions of ${ }^{6-11} \mathrm{Li}$ and the spin-dependent structure functions of ${ }^{7,9,11} \mathrm{Li}$, and study those structure functions in detail. Measurements of those unpolarized and polarized structure functions could be performed by the MUSES project proposed at RIKEN [2] and/or other nuclear facilities in the future [13, 28]. The outline of the present paper is as follows. In Sec. [1], we summarize the main features of the lithium isotopes measured in experiments, and calculate their wave functions using Hartree approximation. The spin-average structure functions of Li are presented in Sec. III. Moreover, we discuss how the neutron structure function can be extracted from Li nuclei. The present way will provide a new alternative to study the neutron structure function using radioactive ions rich in neutrons. In Sec. $\mathbb{\nabla}$ we calculate the spin structure functions of the ${ }^{7,9,11} \mathrm{Li}$ isotopes. The multipole spin structure functions of ${ }_{1}^{3 / 2} g_{1}$ and ${ }_{3}^{3 / 2} g_{1}$ for a spin-3/2 target are discussed in detail. The effect of the nuclear binding and Fermi motion on ${ }_{1}^{3 / 2} g_{1}$ is less than $10 \%$ for $x<0.7$, but it becomes important at large $x$, as in the EMC effect. The spin structure function of ${ }_{3}^{3 / 2} g_{1}$ is novel and it first arises for a spin-3/2 target. It is negative for small $x$ but it turns to be positive for $0.2<x<0.5$. For large $x$ it is again negative and the magnitude becomes large because of the Fermi motion. We summarize our results and discuss several candidates for testing the Gottfried and Bjorken sum rules in mirror nuclei in the last section. 


\section{STRUCTURE OF LI ISOTOPES}

We show the properties of the ${ }^{6-11} \mathrm{Li}$ isotopes in Table \&. It is quite remarkable that the two-neutron separation energy, $S_{2 n}$, decreases dramatically as the atomic mass number, $A$ $=Z+N$, increases and that at the neutron drip line $S_{2 n}$ of ${ }^{11} \mathrm{Li}$ reaches about $300 \mathrm{keV}$. The one-neutron separation energy, $S_{n}$, also diminishes with increasing $A$ (although it shows a dependence on the shell structure), while the one-proton separation energy, $S_{p}$, increases, mainly due to the attractive $p$ - $n$ interaction in nuclei. It is also notable that the rms radius, $r_{A}$, of ${ }^{11} \mathrm{Li}$ is about $3.1 \mathrm{fm}$, which is significantly larger than $A^{1 / 3} \sim 2.2 \mathrm{fm}$. Furthermore, we list the energy levels of the first $1 / 2^{-}$states of ${ }^{7} \mathrm{Li},{ }^{7} \mathrm{Be},{ }^{9} \mathrm{Li}$, and ${ }^{9} \mathrm{Be}$ in Table 1 田, 5], as these are expected to provide information on the spin-orbit force for the nucleons in the $p$-shell.

\section{A. Model for Li isotopes}

During the last 15 years the structure of light $\beta$-unstable nuclei has been studied intensively. In particular, ${ }^{11} \mathrm{Li}$, which is located at the neutron drip line, has attracted a lot of attention. There two valence neutrons are so weakly bound to ${ }^{9} \mathrm{Li}$ that they form a halo structure. From this point of view, some simple cluster models such as the di-neutron cluster model [29], where the valence neutrons are correlated strongly enough to be regarded as one particle, were first proposed, and those simple models succeeded in reproducing the qualitative features of ${ }^{11} \mathrm{Li}$. With increasing observed data on ${ }^{11} \mathrm{Li}$, it was, however, realized that these simple cluster models could not explain the measured experimental data. Thus, some authors started calculating the properties of ${ }^{11} \mathrm{Li}$ using a three-body model [30], which was supported by the fact that ${ }^{11} \mathrm{Li}$ is a Borromean system $\left({ }^{11} \mathrm{Li}={ }^{9} \mathrm{Li}+n+n\right)$, where all the two-body sub-systems are unbound. At present the three-body model is one of the best ways to describe the observed properties of ${ }^{11} \mathrm{Li}$. However, speaking strictly, the three-body model is only valid for structureless particles. In this sense a standard model to describe ${ }^{11} \mathrm{Li}$ has not yet been established.

Our aim in the present paper is to investigate the nuclear structure functions of the $\mathrm{Li}$ isotopes and, in particular, to determine the momentum distribution of each nucleon in these nuclei. Thus, the three-body model is not suitable for our purpose. In addition to this, since we consider that the separation energy is more significant for calculating nuclear structure functions than the other properties of a nucleus, in the present paper we use a simple shell model with Hartree approximation to calculate single-particle wave functions of Li isotopes. Note that it implies that the valence neutrons correlate rather weakly in the halo of ${ }^{11} \mathrm{Li}$. In subsection IIC, we will discuss effects of residual two-body interactions.

In Hartree approximation the total Hamiltonian of a $A$-nucleon system is given in terms of a sum of nucleon kinetic energy and an effective one-body potential, $U$ :

$$
H=\sum_{i=1}^{A}\left[-\frac{\hbar^{2}}{2 M} \vec{\nabla}_{i}^{2}+U\left(\xi_{i}\right)\right],
$$

where $M(=939 \mathrm{MeV})$ is the nucleon mass, and $\xi_{i}$ denotes position $\vec{r}_{i}$, spin $\vec{\sigma}_{i}$, and isospin

$\vec{\tau}_{i}$ of the $i$-th nucleon. The $A$-body wave function, $\Psi\left(\xi_{1}, \cdots, \xi_{i}, \cdots \xi_{A}\right)$, is then given by 
the product of single-particle wave functions, $\phi_{\alpha_{i}}\left(\xi_{i}\right)\left(\alpha_{i}\right.$ stands for all quantum numbers associated with the $i$-th nucleon). In this model the single-particle separation energy is simply given by $-\varepsilon_{i}$.

Determining the consistent isoscalar and isovector mean field potentials is very important for discussing not only neutron-rich nuclei but also neutron stars [31]. However, it would need several elaborate experimantal and theoretical works to fix both the potentials uniquely. In the present paper, therefore, we suppose as a simple and effective potential as possible. It is spherical and consisits of the nuclear, $U_{N}$, and Coulomb, $U_{C}$, parts:

$$
U\left(A, Z, \tau_{z} ; r\right)=U_{N}\left(A, Z, \tau_{z} ; r\right)+U_{C}(A, Z ; r) \frac{1-\tau_{z}}{2}
$$

where $\tau_{z}$ is the $z$-component of isospin $\left(\tau_{z}=+1\right.$ for neutron $(n)$ and -1 for proton $\left.(p)\right)$. Here the nuclear part is given by [32]

$$
U_{N}\left(A, Z, \tau_{z} ; r\right)=\frac{-U_{0}}{1+\exp \left(\frac{r-R}{a}\right)}+r_{0}^{2}(\vec{\ell} \cdot \vec{s}) \frac{1}{r} \frac{d}{d r}\left[\frac{-U_{L S}}{1+\exp \left(\frac{r-R}{a}\right)}\right],
$$

with $R=r_{0} \cdot(A-1)^{1 / 3}$. In Eq. (10) the first and second terms are the central and spinorbit forces, respectively, and we further suppose that the potential depths, $U_{0}$ and $U_{L S}$, depend on $A, Z$, and $\tau_{z}$. The geometrical parameters, $r_{0}$ and $a$, are taken to be common to both the protons and neutrons, in order to reduce the number of parameters for the singleparticle potential. The last assumption is also based on the fact that the ${ }^{6-9} \mathrm{Li}$ isotopes are considered to have the normal density distribution (see Table 2 and Fig. 1 of Ref. [5]). (As will be shown in Fig. 1, however, the present potentials yield decoupled proton- and neutrondensity distributions in ${ }^{8} \mathrm{Li}$ and ${ }^{9} \mathrm{Li}$ nuclei. We will discuss the reason for this discrepancy in subsection П1Т.)

Concerning the Coulomb part, we assume that it is formed by a Gaussian charge distribution with the charge of $Z-1$ and a radius parameter $a_{c}$,

$$
\rho_{c}(r)=(Z-1) e \cdot\left(a_{c} \sqrt{\pi}\right)^{-3} e^{-\left(r / a_{c}\right)^{2}} .
$$

We then obtain

$$
U_{C}(r)=\frac{(Z-1) e^{2}}{r} \operatorname{erf}\left(\frac{r}{a_{c}}\right)
$$

where $\operatorname{erf}(x)$ is the error function [33] and $a_{c}$ is determined from the rms radius of the Gaussian distribution

$$
\left\langle r^{2}\right\rangle_{C}=\frac{3}{2} a_{c}^{2}
$$

We here use the experimental rms radii of the matter distributions of the Li nuclei except for ${ }^{11} \mathrm{Li}$. For ${ }^{11} \mathrm{Li}$, we use the matter distribution of ${ }^{9} \mathrm{Li}$ as the rms radius of the charge distribution. 


\section{B. Shell configuration}

Assuming that identical nucleons in a given shell orbit are coupled to a spin-singlet state and that no two-body residual forces exist, we can fix the configurations for the ground states in the Li isotopes, based on the simple shell model. For example, one can write the shell configuration for ${ }^{9} \mathrm{Li}$ as

$$
\left(\pi 1 s_{1 / 2}\right)^{2}\left(\pi 1 p_{3 / 2}\right)^{1}\left(\nu 1 s_{1 / 2}\right)^{2}\left(\nu 1 p_{3 / 2}\right)^{4}
$$

We also assume that the valence neutrons in ${ }^{11} \mathrm{Li}$ occupy the $\nu 1 p_{1 / 2}$ orbit although higher configurations such as $1 d_{5 / 2}$ and $2 s_{1 / 2}$ are thought to be involved in forming a realistic halo structure. In our model the single-particle excitation energies of a neutron and proton are given by

$$
\Delta \varepsilon(n)=\left|\varepsilon_{n}\left(1 p_{1 / 2}\right)-\varepsilon_{n}\left(1 p_{3 / 2}\right)\right|, \quad \Delta \varepsilon(p)=\left|\varepsilon_{p}\left(1 p_{1 / 2}\right)-\varepsilon_{p}\left(1 p_{3 / 2}\right)\right|,
$$

respectively, and the rms radii of ${ }^{6-9} \mathrm{Li}$ and ${ }^{11} \mathrm{Li}$ nuclei are given by

$$
\begin{aligned}
r_{A=6-9}^{2} & =\frac{1}{A}\left(2\left\langle r^{2}\right\rangle_{\pi 1 s_{1 / 2}}+2\left\langle r^{2}\right\rangle_{\nu 1 s_{1 / 2}}+\left\langle r^{2}\right\rangle_{\pi 1 p_{3 / 2}}+(A-5)\left\langle r^{2}\right\rangle_{\nu 1 p_{3 / 2}}\right), \\
r_{A=11}^{2} & =\frac{1}{A}\left(2\left\langle r^{2}\right\rangle_{\pi 1 s_{1 / 2}}+2\left\langle r^{2}\right\rangle_{\nu 1 s_{1 / 2}}+\left\langle r^{2}\right\rangle_{\pi 1 p_{3 / 2}}+4\left\langle r^{2}\right\rangle_{\nu 1 p_{3 / 2}}+2\left\langle r^{2}\right\rangle_{\nu 1 p_{1 / 2}}\right),
\end{aligned}
$$

respectively.

\section{Model parameters and comments}

In order to obtain the potential parameters, we divide the Li isotopes into two groups: one consists of ${ }^{6} \mathrm{Li}$ and ${ }^{7} \mathrm{Li}$, and the other consists of ${ }^{8} \mathrm{Li},{ }^{9} \mathrm{Li}$ and ${ }^{11} \mathrm{Li}$. In the former and latter groups we start with ${ }^{7} \mathrm{Li}$ and ${ }^{9} \mathrm{Li}$, respectively. The energy differences between the first $1 / 2^{-}\left(1 p_{1 / 2}\right)$ and ground states $\left(1 p_{3 / 2}\right)$ in ${ }^{7} \mathrm{Li}$ and ${ }^{7} \mathrm{Be}$ are less than $500 \mathrm{keV}$, while those in ${ }^{9} \mathrm{Li}$ and ${ }^{9} \mathrm{Be}$ are less than $3 \mathrm{MeV}$ (see Table III). We therefore assume that the spin-orbit force in ${ }^{7,9} \mathrm{Li}$ is not so strong and that it does not affect much the rms radius and single-particle energy levels. First, for each of ${ }^{7,9} \mathrm{Li}$ we roughly determine four potential parameters, $r_{0}, a$ and $U_{0}$, for $n$ and $p$, so as to produce the rms radius, $r_{A}$, as well as the one-neutron and one-proton separation energies, $S_{n}$ and $S_{p}$, with a constraint that the four parameters obtained are not far from the standard values of the potential parameter set given in Ref. [34]. (Note that the two geometrical parameters, $r_{0}$ and $a$, are common to both $p$ and $n$.)

We next added the spin-orbit potentials, $U_{L S}$, for $n$ and $p$ to fit approximately the energy difference, $\Delta \varepsilon$, between the first excited and ground states. Then, we finally tune all the potential parameters to exactly reproduce $S_{n}, S_{p}, \Delta \varepsilon(p), \Delta \varepsilon(n)$ and $r_{A}$ in the vicinity of the values determined in the rough fitting. For the other nuclei in each group, we start parameter-fitting using the values for ${ }^{7} \mathrm{Li}$ or ${ }^{9} \mathrm{Li}$ and find an appropriate set under the condition that the parameter values differ from those for ${ }^{7} \mathrm{Li}$ or ${ }^{9} \mathrm{Li}$ as little as possible.

We present the optimum parameter set in Table III and show how the present model reproduces the experimental data in Table IV. We have also checked numerically how the 
properties of the Li isotopes depend on the choice of parameter values. It is then found that the nuclear properties depend on $r_{0}$ and $U_{0}$ somewhat strongly, but that its dependence on $a$ and $U_{L S}$ are weak as we expected first.

We comment here on the one-neutron separation energy of ${ }^{11} \mathrm{Li}$ in Table III. The value of $S_{n}$ obtained from the experimental binding energy is $0.326 \pm 0.042 \mathrm{MeV}$, which is larger than its two-neutron separation energy, $0.301 \pm 0.029 \mathrm{MeV}$ (see Table 『). Therefore, the probability for two-neutron removal may be larger than that for removing one-neutron. (Note that ${ }^{10} \mathrm{Li}$ is observed experimentally as a resonance state.) Thus, in the present calculation we suppose two possibilities for the one-neutron separation energy of ${ }^{11} \mathrm{Li}$ in order to see the dependence of the structure functions on $S_{n}$. We take $S_{n}=301$ or $350 \mathrm{keV}$, and hereafter we call the case of $S_{n}=301(350) \mathrm{keV}^{11} \mathrm{Li}-\mathrm{a}(\mathrm{b})$, respectively.

As the neutron number increases the central potential for neutrons becomes shallow, while that for protons becomes deep (although they show an odd-even mass number effect). This is vital to fit the small separation energy of the valence neutrons as well as the observed rms radii of Li isotopes. Remarkably, the strength of the spin-orbit force for both protons and neutrons grows drastically with increasing $A$. In Table $\nabla$ we present the energy levels of the single-particle states in the $\mathrm{Li}$ isotopes. The rms radius calculated for each singleparticle wave function is also given. According to the weak binding of the valence neutrons in Li, the rms radius of the last neutron becomes large with growing $A$.

Here we should point out some caveats for the present model (see Table $\mathbb{\mathbb { Q }}$ ): (i) In the observed data the two-neutron separation energy, $S_{2 n}$, is not twice the one-neutron separation energy, while the present calculation gives $S_{2 n}=2 S_{n}$. (ii) In the low-lying spectra of ${ }^{6} \mathrm{Li}$ and ${ }^{8} \mathrm{Li}$ their degeneracies are decoupled in the experiments. (iii) The distributions of protons and neutrons in ${ }^{8,9} \mathrm{Li}$ are quite different at large $r$ in the calculation (see also subsection [ID). Those difficulties indicate the necessity of including residual two-body interactions in nuclei. The first defect could be amended by considering the effect of a pairing force between neutrons, while the last one may indicate that a $p-n$ pairing is required to suppress the leakage of neutrons in ${ }^{8,9} \mathrm{Li}$. Note that neutron leakage or shrunkage of the proton distribution influences the nuclear structure functions much less than the separation energy. This fact allows us to use the present parameter sets for their single-particle potentials, where $r_{0}$ and $a$ are common to both $p$ and $n$, in spite of the caveat (iii). Furthermore, since we used effective potentials which reproduce the separation energies of nucleons close to the Fermi surface, and it is hard to measure experimentally the separation energies of deeply bound nucleons, it may not be necessary to be too concerned about the caveats (i) and (ii) in the present calculation.

\section{Nucleon kinetic energy and average binding energy}

In Figs. 1 - 3 we present the wave functions in coordinate space, $u_{\alpha}(r)$, and the radial momentum distribution of a nucleon, $\phi_{\alpha}(k)$, in the Li isotopes. One can see that as the neutron number increases the tail of the neutron wave function in the $1 p$ orbit is longer. Accordingly, the distribution of the $1 p$ neutron becomes sharp in momentum space. As expected, the valence neutrons in the halo of ${ }^{11} \mathrm{Li}$ distribute very broadly in coordinate space and their momentum distributions are quite sharp around $k \sim 0.1 \mathrm{fm}^{-1}$. The density

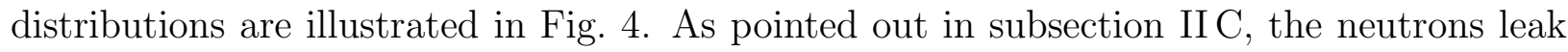


out to large $r$ even in ${ }^{8,9} \mathrm{Li}$, which may imply that a proper (attractive) $p$ - $n$ pairing force is required.

In an unpolarized nucleus the kinetic energy of a nucleon in the single-particle shell, $\alpha=\left\{n, \ell, j, \tau_{z}\right\}$, can be calculated by

$$
\left\langle t_{\alpha}\right\rangle=\frac{1}{2 j+1} \sum_{m} \int \frac{d^{3} k}{(2 \pi)^{3}}\left|\phi_{n \ell j m \tau_{z}}(\vec{k})\right|^{2} \frac{k^{2}}{2 M}=\frac{1}{2 M} \int_{0}^{\infty} \frac{k^{4} d k}{(2 \pi)^{3}}\left|\phi_{\alpha=\left\{n \ell j \tau_{z}\right\}}(k)\right|^{2} .
$$

Here $\phi_{n \ell j m \tau_{z}}(\vec{k})$ is the nucleon momentum distribution, which is given by Fourier transformation of the wave function in coordinate space, and it is normalized as

$$
\frac{1}{(2 \pi)^{3}} \int d^{3} k\left|\phi_{n \ell j m \tau_{z}}(\vec{k})\right|^{2}=1 \text {. }
$$

The kinetic energy of the center-of-mass $(\mathrm{cm})$ motion of the nucleus is then evaluated by

$$
T_{\mathrm{cm}}=\frac{1}{A} \sum_{i}\left\langle t_{\alpha_{i}}\right\rangle
$$

and the spurious cm motion should be subtracted from the total energy. We thus obtain the mean-nucleon kinetic energy, $\langle t\rangle$, in the nucleus and the total binding energy:

$$
\begin{aligned}
\langle t\rangle & =\frac{1}{A}\left(1-\frac{1}{A}\right) \sum_{i}\left\langle t_{\alpha_{i}}\right\rangle, \\
\langle B\rangle & =\frac{1}{2} \sum_{i}\left(\varepsilon_{i}+\left\langle t_{\alpha_{i}}\right\rangle\right)-T_{\mathrm{cm}},
\end{aligned}
$$

respectively. In Table VT we summarize the kinetic energy of the nucleon in each shell model orbit. We should note that the calculated binding energies are rather smaller than the observed ones (see Table [ ). With increasing the neutron number the total binding energy decreases. Probably this discrepancy may be improved by taking residual two-body correlations into account. However, we again emphasize that the present calculation reproduces the observed separation energies of Li isotopes, which plays the most important role in the calculation of nuclear structure functions.

\section{SPIN-AVERAGE STRUCTURE FUNCTIONS OF LI ISOTOPES}

\section{A. Light-cone momentum distributions and nuclear structure functions}

In the DIS of high energy charged leptons off an unpolarized nucleus one can observe the distribution of the longitudinal momentum fraction, $x$, carried by the quarks in the nucleus [8]. The nuclear structure function, $F_{2}^{A}(x)$, is usually described by a convolution of momentum distributions of nucleons in $A$ and the free nucleon structure function [8,9]:

$$
F_{2}^{A}(x)=\sum_{i}^{\mathrm{A}} \int_{x}^{A} d y f_{\alpha_{i} / A}(y) F_{2}^{i}(x / y)
$$


where $F_{2}^{i}=F_{2}^{p(n)}$ when the $i$-th nucleon is a proton (neutron). (Note that in the scaling limit $F_{2}^{A}(x)$ is related to $F_{1}^{A}(x)$ through the Callan-Gross relation. See also Eq. (5).) $f_{\alpha_{i} / A}(y)$ is a distribution of the longitudinal momentum fraction, $y$, carried by the nucleon in the nucleus $A$, which is defined as

$$
0 \leq y / A \equiv \frac{k \cdot q}{P \cdot q} \leq 1 \quad(0 \leq y \leq A)
$$

with $P^{\mu}$ the four momentum of the nucleus $A$.

In the scaling limit the light-cone momentum distribution in the target rest frame is then written as 8 8,9

$$
f_{\alpha_{i} / A}(y)=\int \frac{d^{4} k}{(2 \pi)^{4}} S_{\alpha_{i} / A}(k)\left(1+\frac{k^{3}}{k^{0}}\right) \delta\left(y-\frac{k^{0}+k^{3}}{M}\right)
$$

with the nucleon spectral function

$$
S_{\alpha_{i} / A}(k)=2 \pi \delta\left(k_{0}-M-\varepsilon_{i}+T_{R}\right)\left|\Psi_{\alpha_{i}}(\vec{k})\right|^{2},
$$

where $-\varepsilon_{i}(>0)$ is the separation energy of the $i$-th nucleon in the present approximation, $T_{R}=\vec{k}^{2} / 2 M_{A-1}(>0)$ the recoil energy of the residual nucleus $(A-1)$ with its mass $M_{A-1}$, and $\Psi_{\alpha_{i}}(\vec{k})$ the three-momentum distribution of the struck nucleon $i$. For an unpolarized target, $\left|\Psi_{\alpha_{i}}(\vec{k})\right|^{2}$ is given in terms of the momentum distribution of the nucleon as

$$
\left|\Psi_{\alpha_{i}}(\vec{k})\right|^{2} \equiv \frac{1}{2 j+1} \sum_{m}\left|\phi_{n \ell j m \tau_{z}}(\vec{k})\right|^{2}=\frac{1}{4 \pi}\left|\phi_{\alpha_{i}}(k)\right|^{2},
$$

which is spherically symmetric and independent of the angular variables.

Inserting Eq. (25) into Eq. (24), we find

$$
f_{\alpha_{i} / A}(y)=\int \frac{d^{4} k}{(2 \pi)^{4}} 2 \pi \delta\left(k_{0}-M-\varepsilon_{i}+T_{R}\right)\left|\Psi_{\alpha_{i}}(\vec{k})\right|^{2}\left(1+\frac{k^{3}}{k^{0}}\right) \delta\left(y-\frac{k^{0}+k^{3}}{M}\right) .
$$

Using a new notation

$$
\bar{M}=\bar{M}(k)=M+\varepsilon_{i}-T_{R},
$$

we can rewrite Eq. (27) as

$$
\begin{aligned}
f_{\alpha_{i} / A}(y) & =\int \frac{d^{4} k}{(2 \pi)^{4}} 2 \pi \delta\left(k_{0}-\bar{M}\right)\left|\Psi_{\alpha_{i}}(\vec{k})\right|^{2}\left(1+\frac{k^{3}}{k^{0}}\right) \delta\left(y-\frac{k^{0}+k^{3}}{M}\right) \\
& =\frac{M}{2(2 \pi)^{3}} \int_{0}^{\infty} k d k\left|\phi_{\alpha_{i}}(k)\right|^{2} \int_{-1}^{+1} d \chi\left(1+\frac{k \chi}{\bar{M}}\right) \delta\left(\chi-\frac{M}{k}\left(y-\frac{\bar{M}}{M}\right)\right) .
\end{aligned}
$$

The distribution $f_{\alpha_{i} / A}(y)$ vanishes unless

$$
\left|\frac{M}{k}\left(y-\frac{\bar{M}}{M}\right)\right|<1,
$$


that is,

$$
|M y-\bar{M}|=\left|M y-M-\varepsilon_{i}+T_{R}\right| \leq k .
$$

Since the recoil energy, $T_{R}$, depends on $k^{2}$, Eq. (31) yields a transcendental equation. However, $T_{R}$ is practically small and hence we may replace $T_{R}$ by its expectation value

$$
T_{R} \rightarrow\left\langle T_{R}\right\rangle_{\alpha_{i}} \simeq \frac{\left\langle k^{2}\right\rangle_{\alpha_{i}}}{2 M_{A-1}} \simeq \frac{\left\langle t_{\alpha_{i}}\right\rangle}{A-1} .
$$

Defining the lower limit of the $k$-integral in Eq. (29) as

$$
k_{L} \equiv\left|M y-M-\varepsilon_{i}+\left\langle T_{R}\right\rangle_{\alpha_{i}}\right|
$$

we find

$$
f_{\alpha_{i} / A}(y) \simeq \frac{M^{2}}{\bar{M}} y \int_{k_{L}}^{\infty} \frac{k d k}{2(2 \pi)^{3}}\left|\phi_{\alpha_{i}}(k)\right|^{2} .
$$

The longitudinal momentum distribution, $f_{\alpha_{i} / A}(y)$, must satisfy the condition of baryon number conservation:

$$
\int_{0}^{A} d y f_{\alpha_{i} / A}(y)=1
$$

We have confirmed that $f_{\alpha_{i} / A}(y)$ obtained numerically satisfy the condition given in Eq. (35) with an accuracy of better than $0.2 \%$. In Figs. 5- 7 we present the $f(y)$ distributions for ${ }^{6-11} \mathrm{Li}$. Remarkably, the halo neutrons in ${ }^{11} \mathrm{Li}$ are distributed quite sharply and the shape of $f(y)$ is almost symmetric around $y=1$. This means that to a good approximation such neutrons can be regarded as being essentially free.

\section{B. Numerical results for $F_{2}^{A}$}

Here we present numerical results for the spin-average structure functions of the Li isotopes, which were calculated using Eqs. (22) and (34). For the structure function of the free nucleon, the CTEQ5 parameterization [35] was used. Fig. 8 illustrates the EMC ratios of the Li isotopes, which are defined by $\left(F_{2}^{A}(x) / A\right) /\left(F_{2}^{d}(x) / 2\right)$. We should note that the nuclear structure functions in the EMC ratio are usually corrected to be iso-scalar the so-called iso-scalarity correction [8,9]. However, we did not apply the correction to the present ratio, and hence the Li structure functions in the numerator are not iso-scalar except for ${ }^{6} \mathrm{Li}$. The structure function of the deuteron was calculated using the Paris potential [17]. In order to evaluate its recoil energy, $\left\langle T_{R}\right\rangle$, we employed relativistic kinematics, since the residual debris is just one nucleon. We find that $\left\langle T_{R}\right\rangle \simeq 9.0 \mathrm{MeV}$ and $-\varepsilon+\left\langle T_{R}\right\rangle \simeq 11$ $\mathrm{MeV}$ [36]. (If one chooses nonrelativistic kinematics $\left\langle T_{R}\right\rangle$ is about $9.4 \mathrm{MeV}$ [36].)

In Fig. 8 each EMC ratio increases rapidly with $x$ in the region $x \geq 0.7$ and has a minimum at around $x=0.6$. Also the minimum grows deeper as the number of neutrons increases. The rapid increase of the EMC ratio is caused by the Fermi motion, which can enable the nuclear structure function to have a tiny but finite component at $x>1$. 
Concerning the depression from unity appearing at intermediate $x$, one needs a careful discussion because no iso-scalarity correction is applied in the present EMC ratio.

For ${ }^{6} \mathrm{Li}$, the depression from unity around $x=0.6$ is rather small. From Tables $\nabla$ and $\nabla \mathbb{Z}$ one can calculate the average-separation energies of protons, neutrons and all the nucleons, as well as the average-recoil energy for each Li isotope. They are presented in Table VIT. The average proton- and neutron-separation energies, $\langle\varepsilon\rangle_{p}$ and $\langle\varepsilon\rangle_{n}$, of ${ }^{6} \mathrm{Li}$ are about 15 $\mathrm{MeV}$ and $17 \mathrm{MeV}$, respectively, while the average-recoil energy $\left\langle T_{R}\right\rangle$ is about $2.3 \mathrm{MeV}$. The nuclear binding effect, which is given by the sum of them, is thus about $18 \mathrm{MeV}$ for ${ }^{6} \mathrm{Li}$, while it is about $11 \mathrm{MeV}$ for the deuteron. The small difference between these values is the reason for the small depression in the EMC ratio of ${ }^{6} \mathrm{Li}$.

For $\beta$-unstable $\mathrm{Li}$ isotopes, which are not iso-scalar, the minima appearing around $x=0.6$ in the EMC ratios become deeper as the neutron number increases. Let us consider the case of ${ }^{11} \mathrm{Li}$ as an example. From Table VII we find $\langle\varepsilon\rangle_{n}=6.0(5.9) \mathrm{MeV},\langle\varepsilon\rangle_{p}=30(28) \mathrm{MeV}$, the average-separation energy of the total system, $\langle\varepsilon\rangle_{t o t} \equiv \frac{1}{A}\left(N\langle\varepsilon\rangle_{n}+Z\langle\varepsilon\rangle_{p}\right)=12(12) \mathrm{MeV}$, and $\left\langle T_{R}\right\rangle=1.4$ (1.3) MeV for ${ }^{11} \mathrm{Li}-\mathrm{a}(\mathrm{b})$. Thus, the nuclear binding of ${ }^{11} \mathrm{Li}$ is about $13 \mathrm{MeV}$, which is much closer to that of the deuteron, compared with the case of ${ }^{6} \mathrm{Li}$. It is then expected that the depression in the EMC ratio of ${ }^{11} \mathrm{Li}$ would be more suppressed than that of ${ }^{6} \mathrm{Li}$. However, the EMC ratio shown in Fig. 8 is completely opposite. Since $\beta$-unstable $\mathrm{Li}$ isotopes have more neutrons than protons, as the neutron number increases the neutron structure function should contribute more to the EMC ratio than does the proton.

Let us roughly estimate the effect of neutron excess on the EMC ratio. We suppose that a nucleus is a simple collection of protons and neutrons and that the binding effect is ignored. It is well known that $F_{2}^{p}(x)>F_{2}^{n}(x)$ and around $x=0.6$ the structure function of the free neutron is about a half of that of the free proton [37]. In this case the EMC ratio of ${ }^{6} \mathrm{Li}$ to the deuteron is just unity because ${ }^{6} \mathrm{Li}$ is iso-scalar. Using the relationship $F_{2}^{p}(x) \simeq 2 F_{2}^{n}(x)$ one finds that the ratios for ${ }^{7,8,9,11} \mathrm{Li}$ become $0.95,0.92,0.89$ and 0.85 at $x \simeq 0.6$, respectively. It is remarkable that those values are already close to the calculated ratios at $x=0.6$ in the figure. When the effects of the nuclear binding and Fermi motion are turned on, they, of course, suppress the Li structure function at intermediate $x$ and enhance it at large $x$. However, such a suppression at intermediate $x$ may not be large because it is approximately determined by the difference between the sum of the average separation and recoil energies of Li and that of the deuteron - see Table VII.

If those successive EMC ratios could be measured in the future one would obtain systematic information on the off-mass shell effect on the neutron structure function in a nuclear medium [14. We also note that the EMC ratio of ${ }^{11} \mathrm{Li}$ is not sensitive to the choice of the one-neutron separation energy, $S_{n}$ (see Fig. 8).

We next discuss how the last neutron in each Li isotope is bound as compared with the neutron in the deuteron using the following ratio [7]

$$
R_{d}(A)=\frac{F_{2}\left({ }^{A} \mathrm{Li}\right)-F_{2}\left({ }^{A-1} \mathrm{Li}\right)}{F_{2}^{d}-F_{2}^{p}},
$$

where $F_{2}\left({ }^{A} \mathrm{Li}\right)$ is the nuclear structure function of ${ }^{A} \mathrm{Li}$. Note that for $R_{d}(11)$ take the numerator to be $\left[F_{2}\left({ }^{11} \mathrm{Li}\right)-F_{2}\left({ }^{9} \mathrm{Li}\right)\right] / 2$. The results are plotted in Fig. 9. In the region $x<0.7$ the ratios $R_{d}(A)$ exceed unity, except for $A=7$. This behavior may reflect the tendency for the energy level of the neutron- $1 p_{3 / 2}$ orbit to decrease with increasing neutron 
number (see Table $\mathrm{V}$ ), even though the odd-even mass number effect can be seen in those energy levels. In large $x$ region $R_{d}(A)$ becomes less than unity, which is consistent with the fact that the momentum distribution of the last neutron in the $\mathrm{Li}$ isotope does not have a large high-momentum component. However, one has to keep in mind that the contribution of the nucleons in the core nucleus to the structure function $F_{2}\left({ }^{A} \mathrm{Li}\right)$ is not completely cancelled by $F_{2}\left({ }^{A-1} \mathrm{Li}\right)$ and that there remains an unavoidable contamination of the binding effect on the proton in the deuteron in the denominator.

Let us turn to the discussion of the following ratio [7]

$$
R_{n}(A)=\frac{F_{2}\left({ }^{A} \mathrm{Li}\right)-F_{2}\left({ }^{A-1} \mathrm{Li}\right)}{F_{2}^{n}} .
$$

It would be useful to extract the neutron structure function from data for the series of $\mathrm{Li}$ isotopes. (For $A=11$ we set the same numerator as in $R_{d}(11)$.) If one uses the ratio, $R_{n}(A)$, and the observed structure functions of ${ }^{A} \mathrm{Li}$ and ${ }^{A-1} \mathrm{Li}$, then one can obtain the neutron structure function from $\left[F_{2}\left({ }^{A} \mathrm{Li}\right)-F_{2}\left({ }^{A-1} \mathrm{Li}\right)\right]_{\text {obs. }} / R_{n}(A)$. Figs. 10 and 11 show $R_{n}(A)$ for Li isotopes as well as that for the deuteron, $R_{n}(D)=\left(F_{2}^{d}-F_{2}^{p}\right) / F_{2}^{n}$. In the case that the neutron structure function is assumed to be simply given by $F_{2}^{d}-F_{2}^{p}$, one can see from $R_{n}(D)$ that the correction due to the nuclear binding is about $4 \%$ at around $x=0.5$ and that the effect of Fermi motion is quite large in the region $x>0.7$ (about $10 \%$ at $x=0.7)$.

On the other hand, some combinations of the Li structure functions provide a pretty flat ratio below $x \sim 0.6-0.7$. Within the present model, for example, the ratio $R_{n}(9)$ is almost unity for $x<0.5$ and exceeds unity by only about $2 \%$ at $x=0.6$. We find in Table VII that the average-separation energies as well as the average-recoil energies of ${ }^{8} \mathrm{Li}$ are almost identical to those of ${ }^{9} \mathrm{Li}$. This is the reason why $R_{n}(9)$ is so close to unity at small and intermediate $x$. The ratios $R_{n}(11-a)$ and $R_{n}(11-b)$ are also flat below $x<0.5-0.6$ because of the weak binding of the neutrons in the halo of ${ }^{11} \mathrm{Li}$ (See also Table [III.) The ratios are about 1.03 at $x=0.5$. In particular, in $R_{n}(11-b)$ the deviation from unity, $\left|R_{n}(11-b)-1.0\right|$, is below $3 \%$ for $x<0.75$.

However, in the large $x$ region the ratio increases in $R_{n}(11-a)$, while it decreases in $R_{n}(11-b)$. For the case $S_{n}=301 \mathrm{keV}\left({ }^{11} \mathrm{Li}-\mathrm{a}\right)$ the valence neutrons in ${ }^{11} \mathrm{Li}$ are bound more loosely than those for the case of $S_{n}=350 \mathrm{keV}\left({ }^{11} \mathrm{Li}-\mathrm{b}\right)$. Correspondingly, the rms radii of the neutrons in the $1 p_{1 / 2}$-orbit are, respectively, $4.91 \mathrm{fm}$ and $4.82 \mathrm{fm}$ for the former and latter cases (see Table $\mathrm{\nabla}$ ). In order to reproduce the measured $\mathrm{rms}$ radius of $r_{{ }^{11} \mathrm{Li}}=3.10$ $\mathrm{fm}$, the nucleons in the core need to be more tightly bound for the former case than for the latter (see again Table $\mathrm{V}$ ). Thus, the effect of Fermi motion due to the nucleons in the core appears to be stronger for the former case than that for the latter, which makes $R_{n}(11-a)$ increase and $R_{n}(11-b)$ decrease in the region $x>0.7$. From the behavior of $R_{n}(11)$ at large $x$, we can say that the ratio is quite sensitive to how loosely the neutrons in the halo of ${ }^{11} \mathrm{Li}$ are bound, and that the ratio strongly depends on high momentum components in the nucleon momentum distributions for ${ }^{9} \mathrm{Li}$ and ${ }^{11} \mathrm{Li}$. It is certainly intriguing to work

\footnotetext{
${ }^{1}$ Recall that the cases "11 $\operatorname{Li}(\mathrm{a}-\mathrm{b})$ " correspond to the two choices of separation energy introduced earlier, $S_{n}=301$ (a) or $S_{n}=350$ (b) keV.
} 
out a more elaborate calculation for the nuclear structure of the Li isotopes, where residual interactions such as pairing correlations are taken into account, in order to study the ratio $R_{n}(A)$ in detail.

We have already noticed that the energy spectra of the Li isotopes show the odd-even mass number effect (see Table $\nabla$ ). As a method for cancelling the effect simply, it would be interesting to see the following ratios:

$$
\begin{aligned}
& R_{d 2}(A)=\frac{F_{2}\left({ }^{A} \mathrm{Li}\right)-F_{2}\left({ }^{A-2} \mathrm{Li}\right)}{2\left(F_{2}^{d}-F_{2}^{p}\right)} \\
& R_{n 2}(A)=\frac{F_{2}\left({ }^{A} \mathrm{Li}\right)-F_{2}\left({ }^{A-2} \mathrm{Li}\right)}{2 F_{2}^{n}}
\end{aligned}
$$

Fig. 12 presents the ratios for ${ }^{8} \mathrm{Li}$ and ${ }^{9} \mathrm{Li}$. (Note that $R_{d 2}(A=11)=R_{d}(11)$ and $R_{n 2}(11)=$ $R_{n}(11)$.) One can again see that the ratios for $x<0.6$ are pretty close to unity and the rapid increase due to the Fermi motion appears for $x>0.6$. In particular, the deviation of $R_{d 2}(8)$ $\left(R_{n 2}(9)\right)$ from unity lies within $2 \%(3 \%)$ for $x<0.55$. Interestingly, $R_{d}(9)$ decreases in the large $x$ region, while $R_{d 2}(9)$ increases there. It means that the ${ }^{8} \mathrm{Li}$ nucleus has relatively larger high-momentum components than ${ }^{7} \mathrm{Li}$, which may partly result from the fact that the binding energy of protons in the $1 s_{1 / 2}$ orbit for ${ }^{8} \mathrm{Li}$ is much larger than that for ${ }^{7} \mathrm{Li}$ (see Table (V).

\section{SPIN STRUCTURE FUNCTIONS OF LI ISOTOPES}

Contrary to the case of unpolarized structure functions [8,9], so far, only a few theoretical studies have been made for the effect of nuclear binding on the spin-dependent structure functions [14, 17, 25, 38 42]. The present situation seems to be that the errors in extracting nuclear effects from the spin-dependent structure functions still seem to be within the experimental error bars. However, recent progress in high precision measurements of spindependent structure functions for the neutron, proton, and deuteron [20,23], together with improved parameterizations for the polarized parton distribution functions (PPDF) [43,44], will make it necessary to know the effect with a high accuracy in near future.

\section{A. Nuclear spin structure functions}

Here we systematically consider the spin structure functions of the Li isotopes. Let us suppose that the spin-dependent structure functions of a nucleus separate into the contributions from those of protons and neutrons. In the conventional nuclear model the polarization of a nuclear ground state is determined by the unpaired nucleons because two identical nucleons in the same shell orbit prefer coupling to spin of zero. Thus, the spin of a Li isotope with an odd mass number is $3 / 2$, which is attributed only to the valence proton in the $1 p_{3 / 2}$ orbit, while the spin of the ${ }^{6(8)} \mathrm{Li}$ ground state is $1(2)$, which is given by a combination of the unpaired proton and neutron in the $1 p_{3 / 2}$ orbit (see Table 而).

It is, however, important to consider residual interactions between single-particle states

in a nucleus, which lead the configuration mixing and hence a change of the wave function of 
the valence nucleon. If we ignore the nuclear binding and Fermi motion effects the nuclear spin structure function may be approximately given by [25,40]

$$
g_{1}^{A}(x)=P_{n}^{A} \times g_{1}^{n}(x)+P_{p}^{A} \times g_{1}^{p}(x),
$$

where $P_{p(n)}^{A}$ is the effective polarization of proton (neutron) in the nucleus $A$. It is well known that, for example, in ${ }^{3} \mathrm{He}$ the tensor force etc. provide a mixture of $S^{\prime}$ - and $D$-states to the $S$-wave state and hence that the neutron is somewhat depolarized and the proton gains a slight downward (negative) polarization due to the configuration mixing: $P_{n}^{A=3}=86 \pm 2 \%$ and $P_{p}^{A=3}=2 \times(-2.8 \pm 0.4) \%$ 40. In the series of Li isotopes a similar situation would occur because of a mixing of higher configurations. It is therefore necessary to perform a calculation of the nuclear structure of $\mathrm{Li}$ isotope including residual interactions to get precise values of the effective polarizations. We do not perform such a calculation in this paper.

Instead, in this section we consider the effects of nuclear binding and Fermi motion on the spin asymmetry for a target with spin $3 / 2$ in detail. This corresponds to DIS off ${ }^{7,9,11} \mathrm{Li}$ targets. For such targets there are four non-vanishing multipole structure functions in the scaling limit: ${ }_{0}^{3 / 2} F_{1},{ }_{2}^{3 / 2} F_{1},{ }_{1}^{3 / 2} g_{1}$ and ${ }_{3}^{3 / 2} g_{1}$ - recall the discussion in Sec. [I. The first one is proportional to the usual spin-average structure functions, which has been studied in the previous section, while the second one is analogous to the quadrupole structure functions, $b_{1}$, for a spin-1 target. The last two provide information on quark spin asymmetries: ${ }_{1}^{3 / 2} g_{1}$ is the analog to $g_{1}$ for a spin- $1 / 2$ target, while ${ }_{3}^{3 / 2} g_{1}$ is a new asymmetry function. It is important to keep in mind that in the present approximation only unpaired valence nucleons contribute to the multipole spin structure functions.

\section{B. Spin structure function for a spin-3/2 target}

The spin asymmetry for a spin-3/2 Li target is given by [26]

$$
\frac{d \Delta \sigma^{3 / 2}}{d x d y}=C x\left[1-(1-y)^{2}\right] \sum_{L=1,3}{ }_{L}^{3 / 2} g_{1}(x) \rho_{L}^{3 / 2},
$$

where the factor $C$ is given below Eq. (2). Then, we can calculate the multipole spin structure function using the following convolution form:

$$
{ }_{L}^{3 / 2} g_{1}(x)=\int_{x}^{A} d y \frac{1}{y} g_{L}^{3 / 2}(y) g_{1}^{p}(x / y),
$$

where $g_{L}^{3 / 2}(y)$ is the light-cone spin distribution given by $\operatorname{tr}\left[M_{L}^{3 / 2} g_{1}^{3 / 2}\right]$ - note that $g_{1}^{3 / 2}$ is a diagonal $(2 J+1) \times(2 J+1)$ matrix defined below Eq. (3).

We find $g_{L}^{3 / 2}$ explicitly as

$$
\begin{aligned}
& g_{1}^{3 / 2}(y)=\frac{2 \sqrt{5}}{3} c_{0}(y)-\frac{4}{3 \sqrt{5}} c_{2}(y), \\
& g_{3}^{3 / 2}(y)=-\frac{6}{\sqrt{5}} c_{2}(y),
\end{aligned}
$$


where

$$
\begin{aligned}
c_{L}(y) & =\int \frac{d^{4} k}{(2 \pi)^{4}} 2 \pi \delta\left(k_{0}-\bar{M}\right)\left|\Psi_{1 p_{3 / 2}}^{p}(\vec{k})\right|^{2}\left[1+\frac{k^{3}}{k^{0}}-\frac{\vec{k}^{2}-\left(k^{3}\right)^{2}}{k^{0}\left(k^{0}+M\right)}\right] \\
& \times P_{L}(\cos \theta) \delta\left(y-\frac{k^{0}+k^{3}}{M}\right) .
\end{aligned}
$$

In Eq. (45) the term of the square blacket is the so-called flux factor, which stems from the matrix element of $\gamma^{+} \gamma_{5}$ with respect to the nucleon spinor. In the limit $k^{0} \rightarrow M$ it coinsides with the flux factor given in Refs. [9, 42. Instead of the present flux factor, the factor, $\left(1+k^{3} / k^{0}\right)$, which is the same as that for the $f(y)$ distribution, is often used to

calculate the nuclear spin structure function. However, the difference between two cases is less than $2 \%$ in the $y$ distribution (see Fig. 13 in subsection IVC).

After performing the integrals with respect to $k^{0}$ and the angular variables we finally obtain

$$
\begin{aligned}
& c_{0}(y)=\frac{M^{2}}{\bar{M}} \int_{k_{L}}^{\infty} \frac{k d k}{2(2 \pi)^{3}}\left|\phi_{1 p_{3 / 2}}^{p}(k)\right|^{2}\left[y+\frac{1}{2}(y-1)^{2}-\frac{k^{2}}{2 M^{2}}\right], \\
& c_{2}(y)=\frac{M^{2}}{\bar{M}} \int_{k_{L}}^{\infty} \frac{k d k}{4(2 \pi)^{3}}\left|\phi_{1 p_{3 / 2}}^{p}(k)\right|^{2}\left[3 \frac{M^{2}}{k^{2}}\left(y-\frac{\bar{M}}{M}\right)^{2}-1\right]\left[y+\frac{1}{2}(y-1)^{2}-\frac{k^{2}}{2 M^{2}}\right],
\end{aligned}
$$

where $\phi_{1 p_{3 / 2}}^{p}(k)$ is the radial wave function of the valence proton in momentum space. The term of $\mathcal{O}(\varepsilon / M)$ is neglected in the flux factor because its contribution is very small.

\section{Numerical results for spin structure functions}

In Fig. 13 we plot the distributions, $c_{0}(y)$ and $c_{2}(y)$, for ${ }^{7,9,11} \mathrm{Li}$ isotopes. For comparison, we also present the light-cone momentum distribution, $f(y)$, of the valence proton in the $1 p_{3 / 2}$ orbit. One sees that the shape of the $c_{0}(y)$ distribution is very similar to the $f(y)$ distribution, while that of $c_{2}(y)$ is quite different. Note that the small difference between $c_{0}(y)$ and $f(y)$ is caused by the second and third terms of the flux factor in Eq. (46). It should be noted that $c_{L}(y)$ for ${ }^{11} \mathrm{Li}$ is not sensitive to the choice of the one-neutron separation energy, $S_{n}$, and hence that the difference between the $c_{L}(y)$ distributions for $S_{n}=301$ and $350 \mathrm{keV}$ is quite small.

There is a sum rule for the multipole spin structure function [26]:

$$
\int_{0}^{A} d x x^{n-1}{ }_{L}^{J} g_{1}(x)=0, \quad \text { for } 1 \leq n<L .
$$

This offers a sum rule for $c_{2}(y)$

$$
\int_{0}^{A} d y c_{2}(y)=0
$$

while $c_{0}(y)$ is free from such a constraint. We have checked the sum rule for ${ }_{3}^{3 / 2} g_{1}(n=1)$ numerically, and found that the absolute value of the integral, Eq. (48), is less than 0.0014 
for the ${ }^{7,9,11} \mathrm{Li}$ isotopes, where the calculation was performed in the region $10^{-3} \leq x \leq 2.20$. The sum rule is certainly satisfied within the numerical precision.

In Figs. 14 and 15 we present the multipole spin structure functions, ${ }_{1}^{3 / 2} g_{1}$ and ${ }_{3}^{3 / 2} g_{1}$, for $A=7,9,11$ normalized $g_{1}^{p}$ for the free proton. For the numerical calculation we have used the polarized parton distribution functions (PPDF) parameterized by D. de Florian et al. [43] and M. Glück et al. [44] (denoted by GRSV PPDF). We use one of the options for both, up to the next-to-leading-order (NLO) within the $\overline{\mathrm{MS}}$ scheme, which includes the effects of flavor asymmetries in the proton sea. The calculations are performed at $Q^{2}=10$ $\mathrm{GeV}^{2}$. Since the two parameterizations eventually gave very similar results we show only the result with the GRSV parameterization in the figures. Moreover, since the choice of the one-neutron separation energy, $S_{n}$, in ${ }^{11} \mathrm{Li}$ does not affect the calculated result much, we illustrate only the ratios for ${ }^{11} \mathrm{Li}$-a (i.e., $S_{n}=301 \mathrm{keV}$ ).

In graphing the ratio for ${ }_{1}^{3 / 2} g_{1}$ (see Fig. 14) we have divided out the "geometrical factor" $2 \sqrt{5} / 3$ so the plotted ratio would be unity in the absence of the nuclear binding and Fermi motion. The shape of the ratio is very similar to the usual EMC ratio - see also the distributions of $f(y)$ and $c_{0}(y)$ in Fig. 13. The effect of the nuclear binding and Fermi motion on ${ }_{1}^{3 / 2} g_{1}$ is then about $10 \%$ for $x<0.7$, while the Fermi motion effect is very important for large $x$. This observation is consistent with the earlier results for the deuteron 41,42, and ${ }^{3} \mathrm{He}$ 40. For $x<0.6$ the ratio for ${ }^{7} \mathrm{Li}$ is a little shallower than that for ${ }^{9,11} \mathrm{Li}$. This is because the weak binding of the valence proton in ${ }^{7} \mathrm{Li}$ as compared with in ${ }^{9,11} \mathrm{Li}$ isotopes see Table [V.

The shape of the ratio for ${ }_{3}^{3 / 2} g_{1}$ is quite different from that for ${ }_{1}^{3 / 2} g_{1}$. It is negative for small $x$ but it becomes positive for $0.2<x<0.5$. However, the magnitude is very small in the region of $x<0.6$. For very large $x$ it is again negative and its absolute value becomes large because of the Fermi motion. Because of the weaker binding of the valence proton in ${ }^{7} \mathrm{Li}$ than in ${ }^{9,11} \mathrm{Li}$, the magnitude of the ratio for ${ }^{7} \mathrm{Li}$ for $x>0.5$ is smaller than that for ${ }^{9,11} \mathrm{Li}$. We should note here that the present result is quite different from that of Jaffe and Manohar [26]. In particular, the ratio for large $x$ is negative in our realistic calculation, while it is large and positive in Ref. [26]. This discrepancy is caused by an approximation used in Ref. [26], where the distributions of $c_{0}(y)$ and $c_{2}(y)$ are expanded in terms of $\delta$-functions. This amounts to neglecting the positive wings of $c_{2}(y)$ in Fig. 13, which is numerically inaccurate.

\section{SUMMARY AND CONCLUSION}

We have studied the spin-average and spin-dependent structure functions of the lithium isotopes. First, we have calculated the wave functions within Hartree approximation. In the present calculation we used a phenomenological nuclear potential of the Woods-Saxon type, in which parameters are fitted so as to reproduce the separation energies of protons and neutrons, the single-particle excitation energies and the rms radius for each Li isotope. In the calculation, for ${ }^{11} \mathrm{Li}$ the separation energy for the last neutron in the halo was taken to be either $S_{n}=301$ or $350 \mathrm{keV}$ in order to examine the $S_{n}$-dependence of the structure function. Next, using the wave functions we have individually calculated the light-cone momentum distribution of the nucleon in each shell model orbit. We have found that the $f(y)$ distribution of the valence neutron in ${ }^{11} \mathrm{Li}$ is very sharp and almost symmetric around 
$y=1$, which means that this neutron is bound under a condition quite close to the neutron in free space.

Adopting the usual convolution technique [8] we have calculated the spin-average structure functions of Li isotopes. The EMC ratios of the Li nuclei were first illustrated and the role of the neutron structure function in the EMC ratio was discussed. As the neutron number increases the neutron structure function plays a more important role in the nuclear structure function because its weight becomes larger than that of the protons. Furthermore, we have presented the ratio, $R_{d}$, which is defined by the ratio of the difference between the Li structure functions of mass number $A$ and $A-1$ to the difference between the structure functions of the deuteron and the free proton. The ratio, $R_{n}$, which is defined as in $R_{d}$ but the denominator is the free neutron structure function, was also illustrated. The ratios show the sensitivity of the nuclear structure function to the last valence neutron. In particular, the ratios $R_{n}(9)$ and $R_{n}(11)$ are very useful for extracting the free neutron structure function from Li data. We have also investigated the ratios $R_{d 2}$ and $R_{n 2}$, where the numerators are chosen to be $F_{2}\left({ }^{A} \mathrm{Li}\right)-F_{2}\left({ }^{A-2} \mathrm{Li}\right)$, to eliminate the odd-even mass number effect in the $\mathrm{Li}$ structure.

We have studied the spin-dependent structure functions of Li isotopes. In the present study we have concentrated on Li nuclei with odd $A$ and calculated the multipole spin structure functions, ${ }_{1}^{3 / 2} g_{1}$ and ${ }_{3}^{3 / 2} g_{1}$, where the former is the analog to $g_{1}^{p}$ for a spin- $1 / 2$ target and the latter is a new spin asymmetry which first arises for a spin-3/2 target. We have studied the nuclear binding and Fermi motion effects on those spin structure functions in detail. Here the spin structure function is assumed to be given by a convolution form of the light-cone spin distribution of the valence proton, which is provided by a combination of $c_{0}(y)$ and $c_{2}(y)$, and the spin structure function of the free proton.

We have found that the shape of the ratio of ${ }_{1}^{3 / 2} g_{1}$ to $g_{1}^{p}$ is very similar to the usual EMC ratio, while the ratio for ${ }_{3}^{3 / 2} g_{1}$ is quite different from the EMC one. It has been confirmed that the effect of the nuclear binding and Fermi motion on ${ }_{1}^{3 / 2} g_{1}$ is about $10 \%$ for $x<0.7$, but that the Fermi motion effect is very important for large $x$. On the other hand, the multipole spin structure function of ${ }_{3}^{3 / 2} g_{1}$ is negative for small $x$ but it turns to be positive for $0.2<x<0.5$. For very large $x$ it is again negative and the magnitude of the ratio becomes large because of the Fermi motion. The present observation contradicts an earlier reported result, where an approximation was used to calculate the distributions of $c_{0}(y)$ and $c_{2}(y)$.

We should note that in both the spin-average and spin-dependent cases a shadowing (and anti-shadowing) correction would be necessary for the structure functions at small $x(<0.2)$. This would require delicated calculation along the line of Refs. [24,25]. For further studies it will also be necessary to include two-body residual correlations in the calculation of the structure of Li.

Here we would like to comment on the modification of the Gottfried and Bjorken integrals in nuclei: the former is defined by an integral of the difference between the spinaverage structure functions of a pair of mirror nuclei (divided by $x$ ) [7, 11, 12, , while the latter is given by an integral of the difference between the spin structure functions of mirror nuclei 24,25]. As mentioned in the introduction, such quantities could provide us significant information on the flavor-nonsinglet parton distributions in a nucleus. Together with the flavor-singlet structure functions, such information is quite important for determining the 
individual isospin distributions in a nucleus separately.

We list several candidates for such pairs of mirror nuclei. The most promising candidate is of course the pair ${ }^{3} \mathrm{He}$ and ${ }^{3} \mathrm{H}$ [7, 11, [2], where both the nuclei are stable under strong interactions. However, this is also the lightest pair, so that the nuclear modification on the structure functions cannot be expected to be large. The pair ${ }^{7} \mathrm{Li}$ and ${ }^{7} \mathrm{Be}$ should also be good because ${ }^{7} \mathrm{Be}$ has a long half-life time (53 days) and ${ }^{7} \mathrm{Li}$ is stable. We thus believe that the Li-Be pair may be the best one for testing the nuclear effects on the Gottfried and Bjorken integrals in a medium. The next candidates would be the pairs ${ }^{11} \mathrm{C}^{11} \mathrm{~B}$ and ${ }^{13} \mathrm{~N}-$ ${ }^{13} \mathrm{C}$, where the half-lives of ${ }^{11} \mathrm{C}$ and ${ }^{13} \mathrm{~N}$ are about 20 and 10 minute, respectively, while ${ }^{11} \mathrm{~B}$ and ${ }^{13} \mathrm{C}$ are stable. For other candidates one can find some pairs, whose half-life times are longer than 1 minutes: ${ }^{14} \mathrm{O}(71 \mathrm{sec})-{ }^{14} \mathrm{C}(5730$ years $),{ }^{15} \mathrm{O}(120 \mathrm{sec})-{ }^{15} \mathrm{~N}$ (stable), and ${ }^{17} \mathrm{~F}$ $(65 \mathrm{sec}){ }^{17} \mathrm{O}$ (stable). However, it is nearly impossible to measure their structure functions with fixed-target experiments.

If DIS with high energy electrons (or muons) off unstable nuclei (like the series of $\mathrm{Li}$ isotopes) is realized by using a collider machine (like MUSES or (Tesla)HERA) in the future [2, 13,28], available mirror pairs would be widely extended and then quark nuclear physics could be developed quite systematically. Although we have discussed only the series of $\mathrm{Li}$ isotopes in this paper, we can point out other interesting radioactive isotopes: for example, the series of isotopes of $\mathrm{Be}, \mathrm{B}, \mathrm{C}, \mathrm{O}, \mathrm{Na}$ and $\mathrm{Mg}$ are of great promise [2]. In particular, the series of oxygen isotopes are interesting. In the series the smallest and largest mass (neutron) numbers are 13 (5) and 24 (16), respectively. For neutron-rich isotopes, a neutron skin or a neutron halo is expected to be formed around the tightly bound ${ }^{16} \mathrm{O}$ core. If we could control the atomic number and the difference between the proton and neutron numbers freely in measuring the nuclear structure functions, it would stimulate a great deal of works that should lead to interesting new quark physics in nuclei [7].

We would like to thank F. Bissey for providing us the light-cone momentum distributions of the deuteron. We also acknowledge numerous valuable discussions with V. Guzey on the polarized structure functions and small $x$ physics. This work was partly supported by the Australian Research Council. M.U. acknowledges support from Fundação de Amparo á Pesquisa do Estado de São Paulo (FAPESP). K.T. would like to acknowledge the hospitality at KFA Jülich, where some of the numerical calculations were carried out. The present calculation for spin-dependent structure functions was performed using the FORTRAN codes for the polarized parton distribution functions by D. de Florian and R. Sassot [43], and M. Glück, E. Reya, M. Stratmann, and W. Vogelesang [44]. 


\section{REFERENCES}

[1] I. Tanihata, Nucl. Phys. A 654 (1999) 235c.

[2] MUSES project at RIKEN, see the RIKEN homepage, http://www.rarf.riken.go.jp.

[3] I. Tanihata et al., Phys. Rev. Lett. 55 (1985) 2676; Phys. Lett. B 160 (1985) 380.

[4] See "Chart of Nuclides" at http://www.dne.bnl.gov/CoN/index.htm;;

"Table of Isotopes", eighth edition;

I. Tanihata, "On the Possible Use of Secondary Radioactive Beams", Treatise on HeavyIon Science, edited by D. Allan Bromley, Plenum Press (N.Y.), vol.8 (1989) p.443.

[5] I. Tanihata et al., Phys. Lett. B 206 (1988) 592.

[6] For example, T. Kobayashi et al., Phys. Rev. Lett. 60 (1988) 2599.

[7] K. Saito, C. Boros, K. Tsushima, F. Bissey, I.R. Afnan, A.W. Thomas, Phys. Lett. B 493 (2000) 288.

[8] M. Arneodo, Phys. Rep. 240 (1994) 301;

D.F. Geesaman, K. Saito, A.W. Thomas, Ann. Rev. Nucl. Part. Sci. 45 (1995) 337.

[9] G. Piller, W. Weise, Phys. Rep. 330 (2000) 1.

[10] S. Kumano, Phys. Rep. 303 (1998) 183;

J. Speth, A.W. Thomas, Adv. in Nucl. Phys. 24 (1998) 83;

R. Vogt, Prog. Part. Nucl. Phys. 45 (2000) S105.

[11] V. Guzey, K. Saito, M. Strikman, A.W. Thomas, K. Tsushima, Phys. Rev. D 64 (2001) 054503.

[12] K. Saito, V. Guzey, K. Tsushima, A.W. Thomas, Phys. Lett. B 517 (2001) 93.

[13] B.M. Sherril, Proc. of Second International Conference on Radioactive Nuclear Beams, edited by Th. Delbar, Belgium (1991).

[14] K. Saito, A.W. Thomas, Nucl. Phys. A 574 (1994) 659; F.M. Steffens, K. Tsushima, A.W. Thomas, K. Saito, Phys. Lett. B 447 (1999) 233.

[15] G.B West, Phys. Lett. B 37 (1971) 509;

W.B. Atwood, G.B. West, Phys. Rev. D 7 (1973) 773.

[16] W. Melnitchouk, A.W. Thomas, Phys. Lett. B 377 (1996) 11.

[17] I.R. Afnan, F. Bissey, J. Gomez, A.T. Katramatou, W. Melnitchouk, G.G. Petratos, A.W. Thomas, Phys. Lett. B 493 (2000) 36.

See also, F. Bissey, A.W. Thomas, I.R. Afnan, Phys. Rev. C 64 (2001) 024004.

[18] E. Pace, G. Salmè, S. Scopetta, A. Kievsky, preprint nucl-th/0109005.

[19] EM Collaboration, J. Ashman et al., Nucl. Phys. B 328 (1989) 1.

[20] For recent reviews of spin structure functions, see, M. Anselmino, A. Efremov, E. Leader, Phys. Rep. 261 (1995) 1;

B. Lampe, E. Reya, Phys. Rep. 332 (2000) 1.

[21] E142 Collaboration, P.L. Anthony et al., Phys. Rev. Lett. 71 (1993) 959.

[22] SM Collaboration, B. Adeva et al., Phys. Lett. B 329 (1994) 399.

[23] E154 Collaboration, K. Abe et al., Phys. Rev. Lett. 79 (1997) 26; Spin Muon Collaboration, B. Adeva et al., Phys. Rev. D 58 (1998) 112001; E143 Collaboration, K. Abe et al., Phys. Rev. D 58 (1998) 112003; HERMES Collaboration, A. Airapetian et al., Phys. Lett. B 442 (1998) 484; E155 Collaboration, P.L. Anthony et al., Phys. Lett. B 458 (1999) 529.

[24] F. Bissey, V. Guzey, M. Strikman, A.W. Thomas, preprint hep-ph/0109069. 
[25] V. Guzey, M. Strikman, Phys. Rev. C 61 (1999) 014002; V. Guzey, Phys. Rev. D 64 (2001) 045201.

[26] R.L. Jaffe, A. Manohar, Nucl. Phys. B 321 (1989) 343.

[27] P. Hoodbhoy, R.L. Jaffe, A. Manohar, Nucl. Phys. B 312 (1988) 571.

[28] See, for example, "The Science Driving the $12 \mathrm{GeV}$ Upgrade of CEBAF", edited by the $12 \mathrm{GeV}$ upgrade white paper steering committee (TJLAB, 2000);

R. Venugopalan (eRHIC project), preprint hep-ph/0102087;

"Future Physics at HERA" at the DESY home page, http://www.desy.de/html/home/index.html.

[29] P. G. Hansen, B. Johnson, Europhys. Lett. 4 (1987) 409.

[30] For reviews, see, M.V. Zhukov, B.V. Danilin, D.V. Fedorov, J.M. Bang, I.J. Thompson, J.S. Vaagen, Phys. Rep. 231 (1993) 151;

E. Garrido, E. Moya de Guerra, Nucl.Phys. A 650 (1999) 387;

E. Garrido, D.V. Fedorov, A.S. Jensen, Phys. Rev. C 59 (1999) 1272.

[31] C.J. Horowitz, J. Piekarewicz, preprint nucl-th/0108036.

[32] A. Bohr, B. R. Mottelson, "Nuclear Structure" vol. 1, World Scientific (Singapore) (1969) p.238.

[33] M. Abramowitz, I. A. Stegun, "Handbook of Mathematical Functions" Dover (New York) (1972) p.297.

[34] A. Bohr, B. R. Mottelson "Nuclear Structure" vol. 1, World Scientific (Singapore), p. 239, Eqs. (2-181) and (2-182).

[35] H.L. Lai et al., Eur. Phys. J. C 12 (2000) 375.

[36] F. Bissey, private communication. See also, Ref. [17].

[37] F. Halzen, A. D. Martin, "Quark \& Leptons: An introductory Course in Modern Particle Physics" (Wiely), Fig. 9.6 (1984) p.200.

[38] F.E. Close, R.G. Roberts, G.G. Ross, Nucl. Phys. B 296 (1988) 582.

[39] D. Indumathi, Phys. Lett. B 374 (1996) 193.

[40] J.L. Friar, B.F. Gibson, G.L. Payne, A.M. Bernstein, T.E. Chupp, Phys. Rev. C 42 (1990) 2310; C. Ciofi degli Atti, S. Scopetta, E. Pace, G. Salmè, Phys. Rev. C 48 (1993) R968.

[41] W. Melnitchouk, G. Piller, A.W. Thomas, Phys. Lett. B 346 (1995) 165.

[42] S. Kulagin, W. Melnitchouk, G. Piller, W. Weise, Phys. Rev. C 52 (1995) 932.

[43] D. de Florian, R. Sassot, Phys. Rev. D 62 (2000) 094025.

[44] M. Glück, E. Reya, M. Stratmann, W. Vogelsang, Phys. Rev. D 63 (2001) 094005. 


\section{TABLES}

TABLE I. Experimental data for Li isotopes [4.5. The one-neutron, $S_{n}(Z, N)$, one-proton, $S_{p}(Z, N)$, and two-neutron, $S_{2 n}(Z, N)$, separation energies of the nucleus ${ }^{A} Z(A=N+Z)$ are defined by $S_{n}(Z, N)=B(Z, N)-B(Z, N-1), S_{p}(Z, N)=B(Z, N)-B(Z-1, N)$ and $S_{2 n}(Z, N)=B(Z, N)-B(Z, N-2)$, respectively, where $B(Z, N)$ is the total binding energy of the nucleus ${ }^{A} Z$. The rms radius of ${ }^{A} \mathrm{Li}$ is denoted by $r_{A}$. The numbers in the parentheses stand for the error bars of the observed data.

\begin{tabular}{llccccc}
\hline \hline & & & & & & \\
& $J^{\pi} ; T$ & $B(\mathrm{MeV})$ & $S_{n}(\mathrm{MeV})$ & $S_{2 n}(\mathrm{MeV})$ & $S_{p}(\mathrm{MeV})$ & $r_{A}(\mathrm{fm})$ \\
\hline${ }^{6} \mathrm{Li}$ & $1^{+} ; 0$ & 31.995 & 5.664 & & 4.589 & 2.32 \\
& & $(0.0005)$ & $(0.0505)$ & & $(0.0505)$ & $(0.03)$ \\
${ }^{7} \mathrm{Li}$ & $3 / 2^{-} ; 1 / 2$ & 39.245 & 7.250 & 12.91 & 9.998 & 2.33 \\
& & $(0.0005)$ & $(0.0009)$ & $(0.0505)$ & $(0.0015)$ & $(0.03)$ \\
${ }^{8} \mathrm{Li}$ & $2^{+} ; 1$ & 41.277 & 2.033 & 9.283 & 12.45 & 2.37 \\
& & $(0.0005)$ & $(0.0010)$ & $(0.0010)$ & $(0.0305)$ & $(0.03)$ \\
${ }^{9} \mathrm{Li}$ & $3 / 2^{-} ; 3 / 2$ & 45.341 & 4.064 & 6.096 & 13.93 & 2.32 \\
& & $(0.0019)$ & $(0.0024)$ & $(0.0024)$ & $(0.0090)$ & $(0.02)$ \\
${ }^{11} \mathrm{Li}$ & $3 / 2^{-} ; 5 / 2$ & 45.642 & 0.326 & 0.301 & 15.30 & 3.10 \\
& & $(0.0271)$ & $(0.0422)$ & $(0.0290)$ & $(0.0971)$ & $(0.22)$ \\
\hline \hline
\end{tabular}

TABLE II. The first $1 / 2^{-}$levels of ${ }^{7,9} \mathrm{Li}$ and ${ }^{7,9} \mathrm{Be}$ nuclei. The values presented are those of the first excited states (except for ${ }^{9} \mathrm{Be}$ ) and correspond to the single-particle excitations specified in the fouth column. The value for ${ }^{9} \mathrm{Be}$ is for the third excited state. The spins, parities, and energy levels of the first and second excited states of ${ }^{9} \mathrm{Be}$ are $1 / 2^{+}(1.684 \mathrm{MeV})$ and $5 / 2^{-}(2.429 \mathrm{MeV})$, respectively.

\begin{tabular}{lccc}
\hline \hline & $J^{\pi}$ & $E_{X}(\mathrm{MeV})$ & \\
\hline${ }^{7} \mathrm{Li}$ & 0.4776 & $\Delta E\left(\pi 1 p_{1 / 2}-\pi 1 p_{3 / 2}\right)$ \\
${ }^{7} \mathrm{Be}$ & $1 / 2^{-}$ & 0.4291 & $\Delta E\left(\nu 1 p_{1 / 2}-\nu 1 p_{3 / 2}\right)$ \\
${ }^{9} \mathrm{Li}$ & $1 / 2^{-}$ & 2.691 & $\Delta E\left(\pi 1 p_{1 / 2}-\pi 1 p_{3 / 2}\right)$ \\
${ }^{9} \mathrm{Be}$ & $1 / 2^{-}$ & 2.780 & $\Delta E\left(\nu 1 p_{1 / 2}-\nu 1 p_{3 / 2}\right)$ \\
\hline \hline
\end{tabular}


TABLE III. Parameters in the effective potentials for Li nuclei. The numbers (those in the parentheses) are for neutrons (protons). We suppose that the one-neutron separation energy of ${ }^{11} \mathrm{Li}$ is 301 or $350 \mathrm{keV}$, which is, respectively, denoted by ${ }^{11} \mathrm{Li}-\mathrm{a}$ or $\mathrm{b}$ in the potential parameters.

\begin{tabular}{cccccc}
\hline \hline & $U_{0}(\mathrm{MeV})$ & $r(\mathrm{fm})$ & $a(\mathrm{fm})$ & $U_{L S}(\mathrm{MeV})$ & $a_{c}(\mathrm{fm})$ \\
\hline${ }^{6} \mathrm{Li}$ & 51.00 & 1.38 & 0.66 & 1.25 & \\
& $(51.36)$ & $(1.38)$ & $(0.66)$ & $(1.32)$ & $(1.894)$ \\
${ }^{7} \mathrm{Li}$ & 50.05 & 1.38 & 0.66 & 1.26 & \\
& $(58.10)$ & $(1.38)$ & $(0.66)$ & $(1.32)$ & $(1.902)$ \\
${ }^{8} \mathrm{Li}$ & 59.30 & 1.00 & 0.53 & 7.27 & \\
& $(88.01)$ & $(1.00)$ & $(0.53)$ & $(9.00)$ & $(1.935)$ \\
${ }^{9} \mathrm{Li}$ & 55.09 & 1.05 & 0.54 & 12.46 & \\
& $(80.15)$ & $(1.05)$ & $(0.54)$ & $(9.00)$ & $(1.894)$ \\
${ }^{11} \mathrm{Li}-\mathrm{a}$ & 43.81 & 1.08 & 0.51 & 12.46 & \\
& $(71.72)$ & $(1.08)$ & $(0.51)$ & $(9.00)$ & $(1.894)$ \\
${ }^{11} \mathrm{Li}-\mathrm{b}$ & 40.38 & 1.14 & 0.51 & 12.46 & \\
& $(66.60)$ & $(1.14)$ & $(0.51)$ & $(9.00)$ & $(1.894)$ \\
\hline \hline & & & &
\end{tabular}

TABLE IV. Comparison of the calculated results to the experimental data for ${ }^{6-11} \mathrm{Li}$ nuclei. The numbers in the parentheses are the corresponding experimental values (see also Tables 1 and 【1).

\begin{tabular}{cccccc}
\hline \hline & $S_{n}(\mathrm{MeV})$ & $S_{p}(\mathrm{MeV})$ & $\Delta \varepsilon(n)(\mathrm{MeV})$ & $\Delta \varepsilon(p)(\mathrm{MeV})$ & $r_{A}(\mathrm{fm})$ \\
\hline${ }^{6} \mathrm{Li}$ & 5.665 & 4.589 & 0.427 & 0.442 & 2.33 \\
& {$[5.664]$} & {$[4.589]$} & {$[-]$} & {$[-]$} & {$[2.32]$} \\
${ }^{7} \mathrm{Li}$ & 7.246 & 10.006 & 0.429 & 0.476 & 2.33 \\
& {$[7.250]$} & {$[9.998]$} & {$[0.4291]$} & {$[0.4776]$} & {$[2.33]$} \\
${ }^{8} \mathrm{Li}$ & 2.033 & 12.449 & 1.397 & 2.643 & 2.37 \\
& {$[2.033]$} & {$[12.45]$} & {$[-]$} & {$[-]$} & {$[2.37]$} \\
${ }^{9} \mathrm{Li}$ & 4.064 & 13.930 & 2.778 & 2.685 & 2.33 \\
& {$[4.064]$} & {$[13.93]$} & {$[2.780]$} & {$[2.691]$} & {$[2.32]$} \\
${ }^{11} \mathrm{Li}-\mathrm{a}$ & 0.301 & 15.305 & 2.550 & 2.643 & 3.10 \\
& {$[0.326]$} & {$[15.30]$} & {$[-]$} & {$[-]$} & {$[3.10]$} \\
${ }^{11} \mathrm{Li}-\mathrm{b}$ & 0.350 & 15.301 & 2.747 & 2.737 & 3.10 \\
& {$[0.326]$} & {$[15.30]$} & {$[-]$} & {$[-]$} & {$[3.10]$} \\
\hline \hline
\end{tabular}


TABLE V. Calculated energy levels of single-particle states and the rms radius of the nucleon. The numbers are for neutrons, while those in the parentheses are for protons. $r_{N}$ stands for the rms radius calculated by the single-particle wave function of the nucleon.

\begin{tabular}{|c|c|c|c|c|c|c|}
\hline & $\begin{array}{c}1 s_{1 / 2} \\
-\varepsilon(\mathrm{MeV}) \\
\end{array}$ & $r_{N}(\mathrm{fm})$ & $\begin{array}{c}1 p_{3 / 2} \\
-\varepsilon(\mathrm{MeV}) \\
\end{array}$ & $r_{N}(\mathrm{fm})$ & $\begin{array}{c}1 p_{1 / 2} \\
-\varepsilon(\mathrm{MeV}) \\
\end{array}$ & $r_{N}(\mathrm{fm})$ \\
\hline \multirow[t]{2}{*}{${ }^{6} \mathrm{Li}$} & 21.92 & 1.93 & 5.665 & 2.91 & 5.237 & 2.95 \\
\hline & $(20.39)$ & (1.96) & $(4.589)$ & $(2.98)$ & $(4.147)$ & $(3.02)$ \\
\hline \multirow[t]{2}{*}{${ }^{7} \mathrm{Li}$} & 23.13 & 1.96 & 7.246 & 2.84 & 6.817 & 2.87 \\
\hline & $(27.11)$ & $(1.89)$ & (10.01) & $(2.65)$ & (9.530) & $(2.67)$ \\
\hline \multirow[t]{2}{*}{${ }^{8} \mathrm{Li}$} & 21.41 & 1.73 & 2.033 & 3.15 & 0.637 & 4.00 \\
\hline & $(38.51)$ & $(1.51)$ & $(12.45)$ & $(2.18)$ & $(9.805)$ & $(2.26)$ \\
\hline \multirow[t]{2}{*}{${ }^{9} \mathrm{Li}$} & 21.94 & 1.79 & 4.064 & 2.84 & 1.286 & 3.54 \\
\hline & $(37.49)$ & $(1.59)$ & (13.93) & $(2.23)$ & (11.25) & $(2.29)$ \\
\hline \multirow[t]{2}{*}{${ }^{11} \mathrm{Li}-\mathrm{a}$} & 17.86 & 1.95 & 2.851 & 3.13 & 0.301 & 4.91 \\
\hline & $(36.55)$ & (1.67) & $(15.30)$ & $(2.27)$ & (12.66) & $(2.32)$ \\
\hline \multirow[t]{2}{*}{${ }^{11} \mathrm{Li}-\mathrm{b}$} & 16.99 & 2.02 & 3.096 & 3.15 & 0.350 & 4.82 \\
\hline & $(34.88)$ & (1.74) & (15.30) & $(2.33)$ & $(12.56)$ & $(2.38)$ \\
\hline
\end{tabular}

TABLE VI. Kinetic energy of a nucleon in each shell orbit. The integration over the wave function in momentum space was performed in the region $0 \leq k \leq 6 \mathrm{fm}^{-1}$.

\begin{tabular}{ccccccc}
\hline \hline & $\begin{array}{c}\nu 1 s_{1 / 2} \\
(\mathrm{MeV})\end{array}$ & $\begin{array}{c}\nu 1 p_{3 / 2} \\
(\mathrm{MeV})\end{array}$ & $\begin{array}{c}\nu 1 p_{1 / 2} \\
(\mathrm{MeV})\end{array}$ & $\begin{array}{c}\pi 1 s_{1 / 2} \\
(\mathrm{MeV})\end{array}$ & $\begin{array}{c}\pi 1 p_{3 / 2} \\
(\mathrm{MeV})\end{array}$ & $\begin{array}{c}\pi 1 p_{1 / 2} \\
(\mathrm{MeV})\end{array}$ \\
\hline${ }^{6} \mathrm{Li}$ & 12.65 & 16.59 & 16.27 & 12.31 & 16.08 & 15.72 \\
${ }^{7} \mathrm{Li}$ & 12.26 & 17.01 & 16.77 & 13.11 & 19.16 & 18.96 \\
${ }^{8} \mathrm{Li}$ & 15.92 & 17.07 & 13.73 & 20.62 & 28.89 & 27.32 \\
${ }^{9} \mathrm{Li}$ & 14.78 & 18.46 & 14.45 & 18.59 & 27.23 & 26.03 \\
${ }^{11} \mathrm{Li}-\mathrm{a}$ & 12.50 & 15.66 & 10.73 & 16.73 & 25.86 & 25.12 \\
${ }^{11} \mathrm{Li}-\mathrm{b}$ & 11.62 & 15.09 & 10.43 & 15.47 & 24.37 & 23.76 \\
\hline \hline
\end{tabular}


TABLE VII. Calculated average-separation and recoil energies for Li isotopes. $\langle\varepsilon\rangle_{p},\langle\varepsilon\rangle_{n}$ and $\langle\varepsilon\rangle_{t o t}\left(=\left(N\langle\varepsilon\rangle_{n}+Z\langle\varepsilon\rangle_{p}\right) / A\right)$ are, respectively, for the average-separation energies of protons, neutrons and the total system. The average-recoil energy is denoted by $\left\langle T_{R}\right\rangle(=\langle t\rangle /(A-1))$. The bottom row is for the deuteron 36 .

\begin{tabular}{ccccc}
\hline \hline & $\langle\varepsilon\rangle_{p}(\mathrm{MeV})$ & $\langle\varepsilon\rangle_{n}(\mathrm{MeV})$ & $\langle\varepsilon\rangle_{\text {tot }}(\mathrm{MeV})$ & $\left\langle T_{R}\right\rangle(\mathrm{MeV})$ \\
\hline${ }^{6} \mathrm{Li}$ & 15.1 & 16.5 & 15.8 & 2.3 \\
${ }^{7} \mathrm{Li}$ & 21.4 & 15.2 & 17.9 & 2.1 \\
${ }^{8} \mathrm{Li}$ & 29.8 & 9.78 & 17.3 & 2.4 \\
${ }^{9} \mathrm{Li}$ & 29.6 & 10.0 & 16.6 & 2.1 \\
${ }^{11} \mathrm{Li}-\mathrm{a}$ & 29.5 & 5.97 & 12.4 & 1.4 \\
${ }^{11} \mathrm{Li}-\mathrm{b}$ & 28.4 & 5.88 & 12.0 & 1.3 \\
$\mathrm{D}$ & - & - & 2.22 & 9.0 \\
\hline \hline
\end{tabular}




\section{FIGURES}
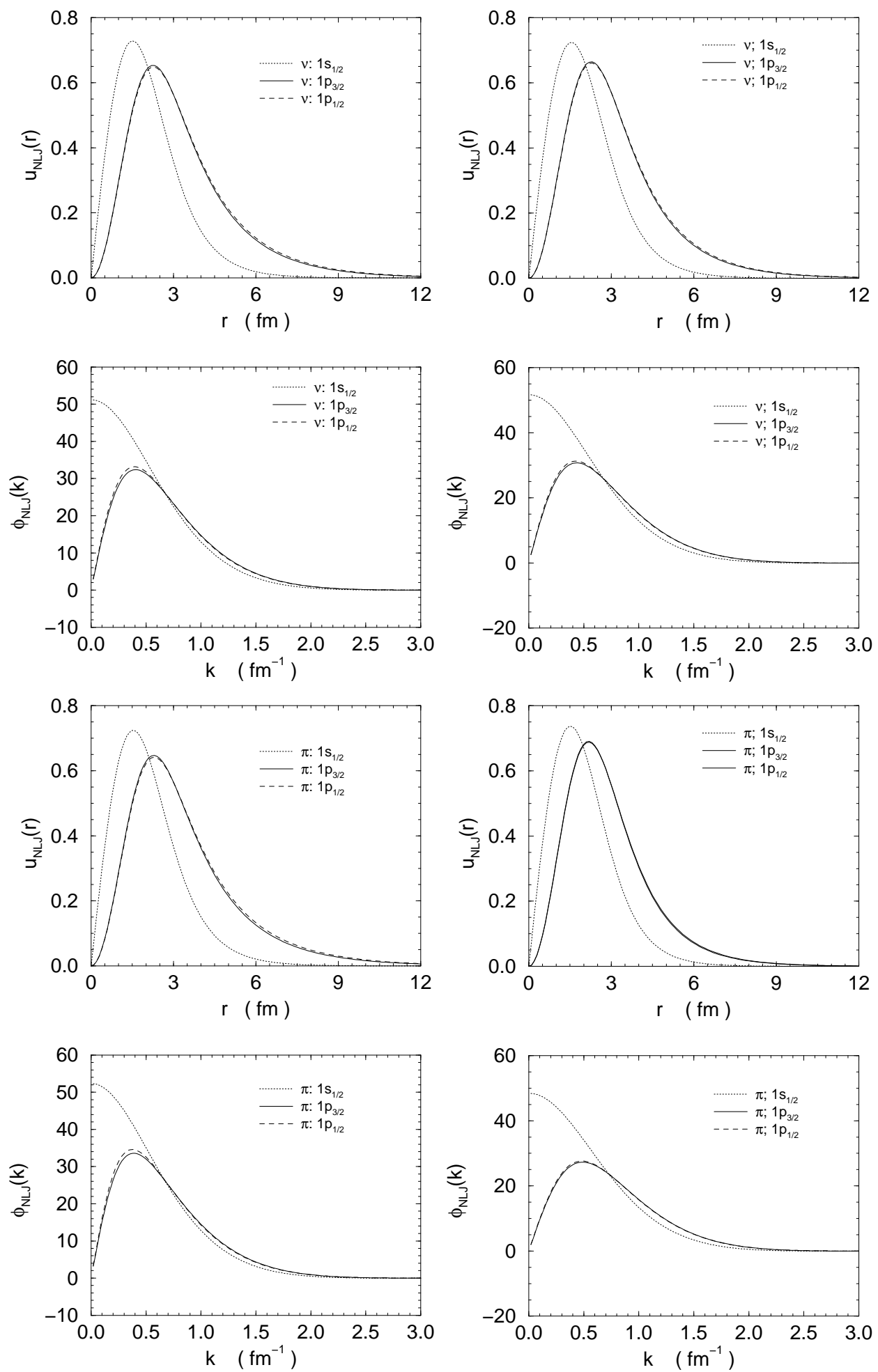

FIG. 1. Radial wave functions, $u_{\alpha}(r)$, and momentum distributions, $\phi_{\alpha}(k)$, of the single-particle states in ${ }^{6} \mathrm{Li}$ (left panels) and ${ }^{7} \mathrm{Li}$ (right panels). The top two panels are for neutrons, while the bottom two are for protons. In each panel the dotted, solid and dashed lines denote the distributions of the nucleons in the $1 s_{1 / 2}, 1 p_{3 / 2}$ and $1 p_{1 / 2}$ shell orbits, respectively. 

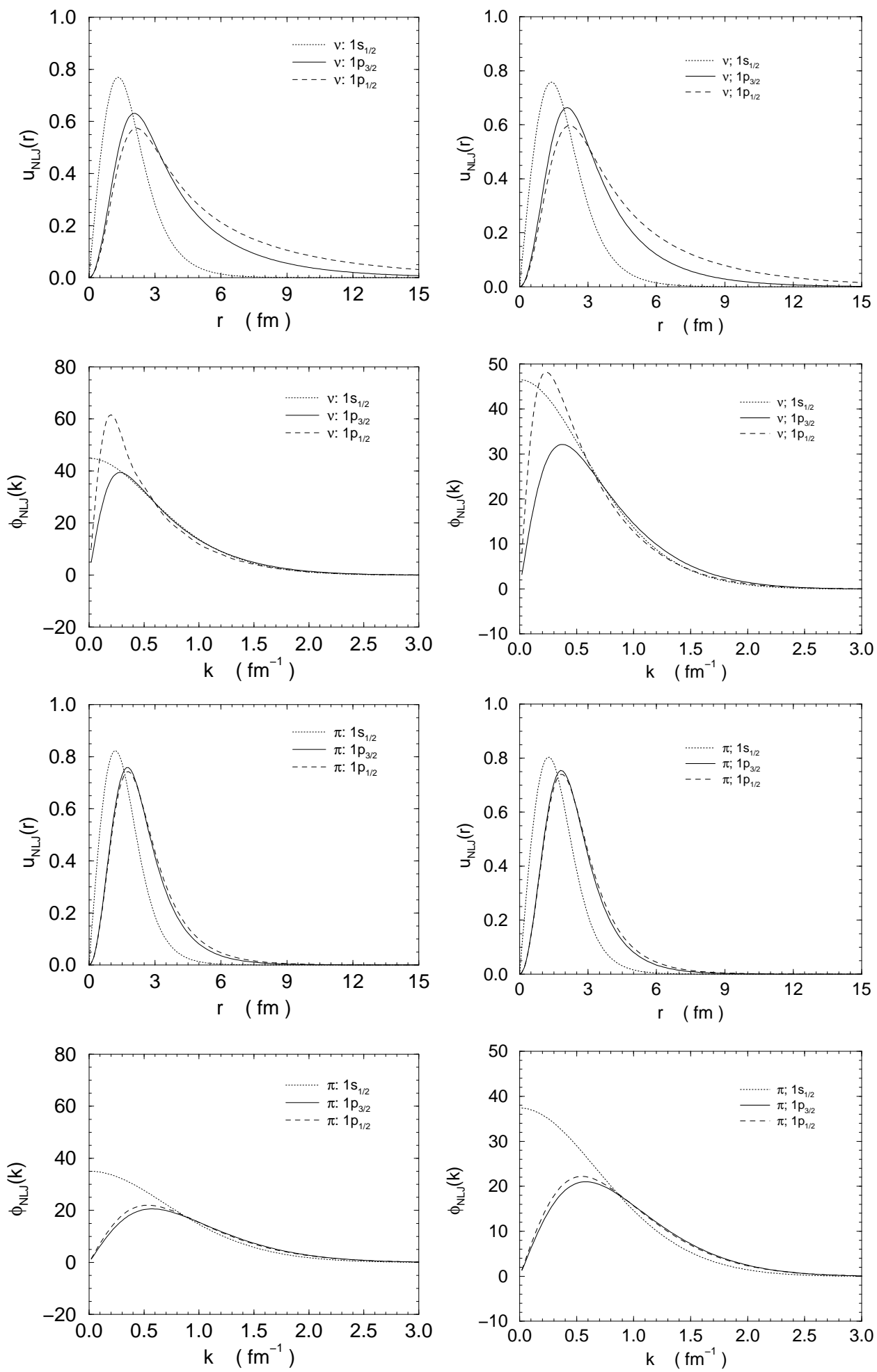

FIG. 2. Same as Fig. 1 but for ${ }^{8} \mathrm{Li}$ (left panels) and ${ }^{9} \mathrm{Li}$ (right panels). 

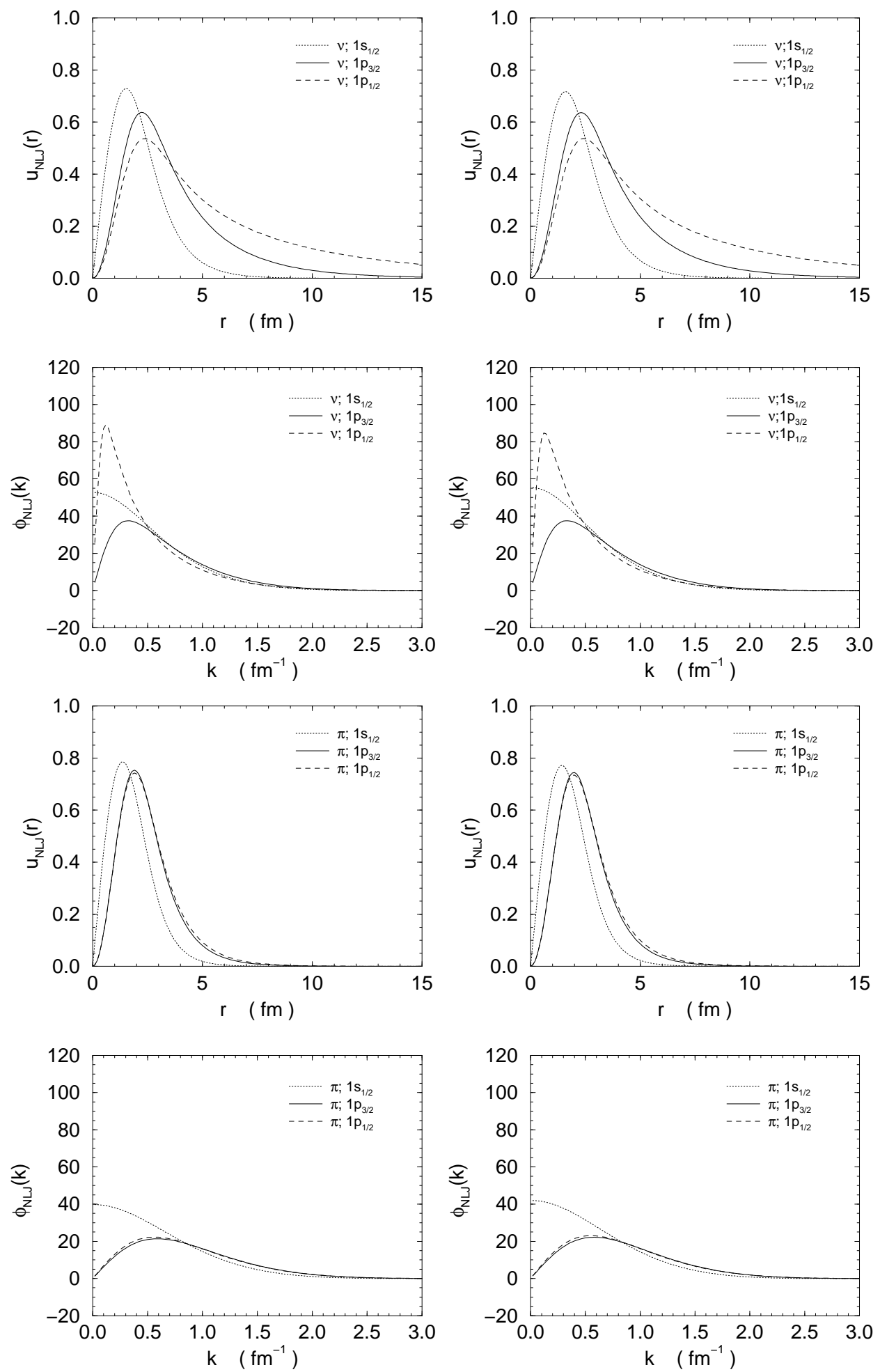

FIG. 3. Same as Fig. 目 but for ${ }^{11} \mathrm{Li}$. The left and right panels are the results for the cases of $S_{n}=301 \mathrm{keV}$ and $350 \mathrm{keV}$, respectively. 

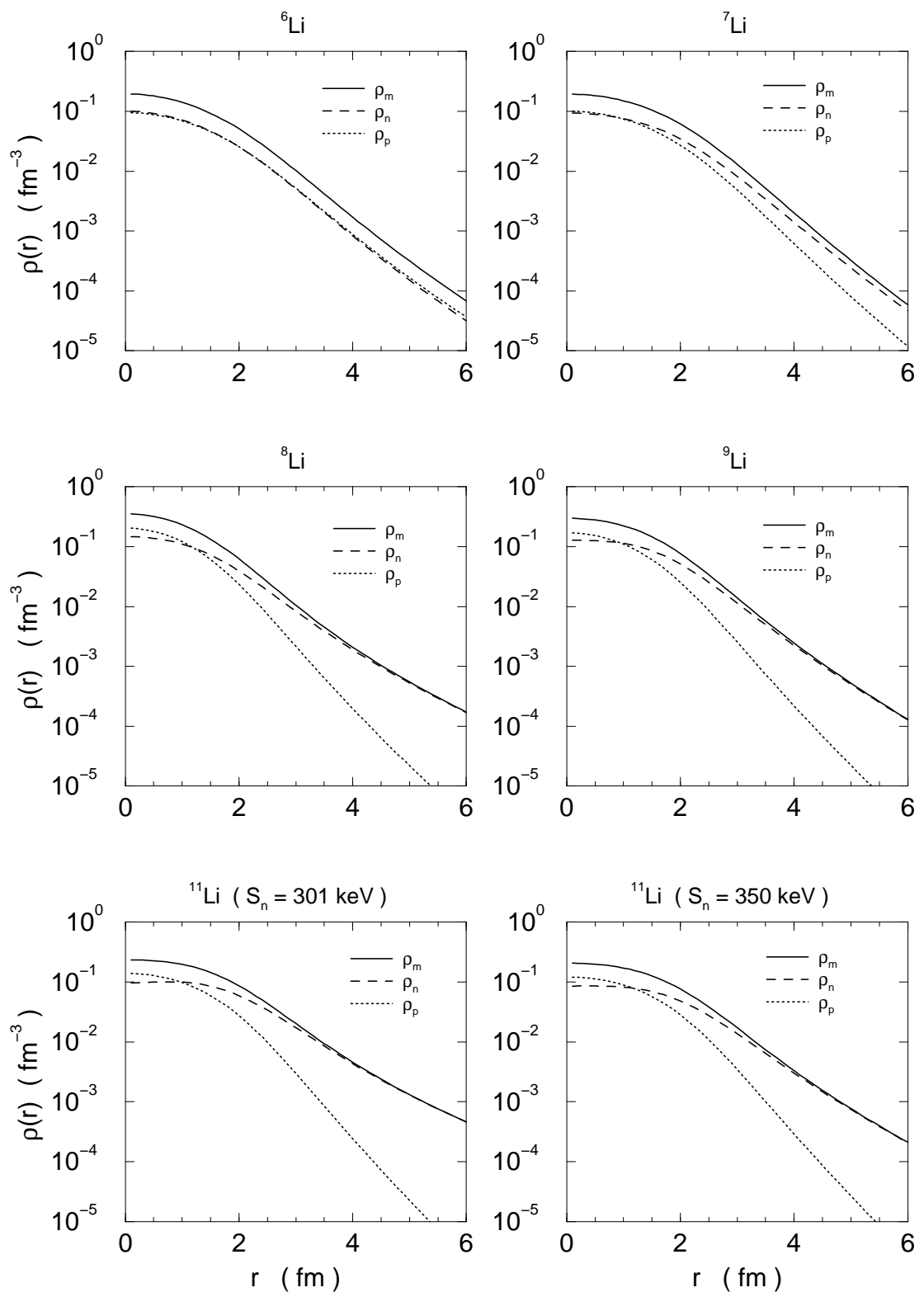

FIG. 4. Matter $\left(\rho_{m}\right)$, neutron $\left(\rho_{n}\right)$ and proton $\left(\rho_{p}\right)$ density distributions in ${ }^{6-11} \mathrm{Li}$. The neutronand proton density distributions are obtained by summing up of the square of the corresponding single-particle wave functions. The matter distribution is simply given by $\rho_{p}+\rho_{n}$. 

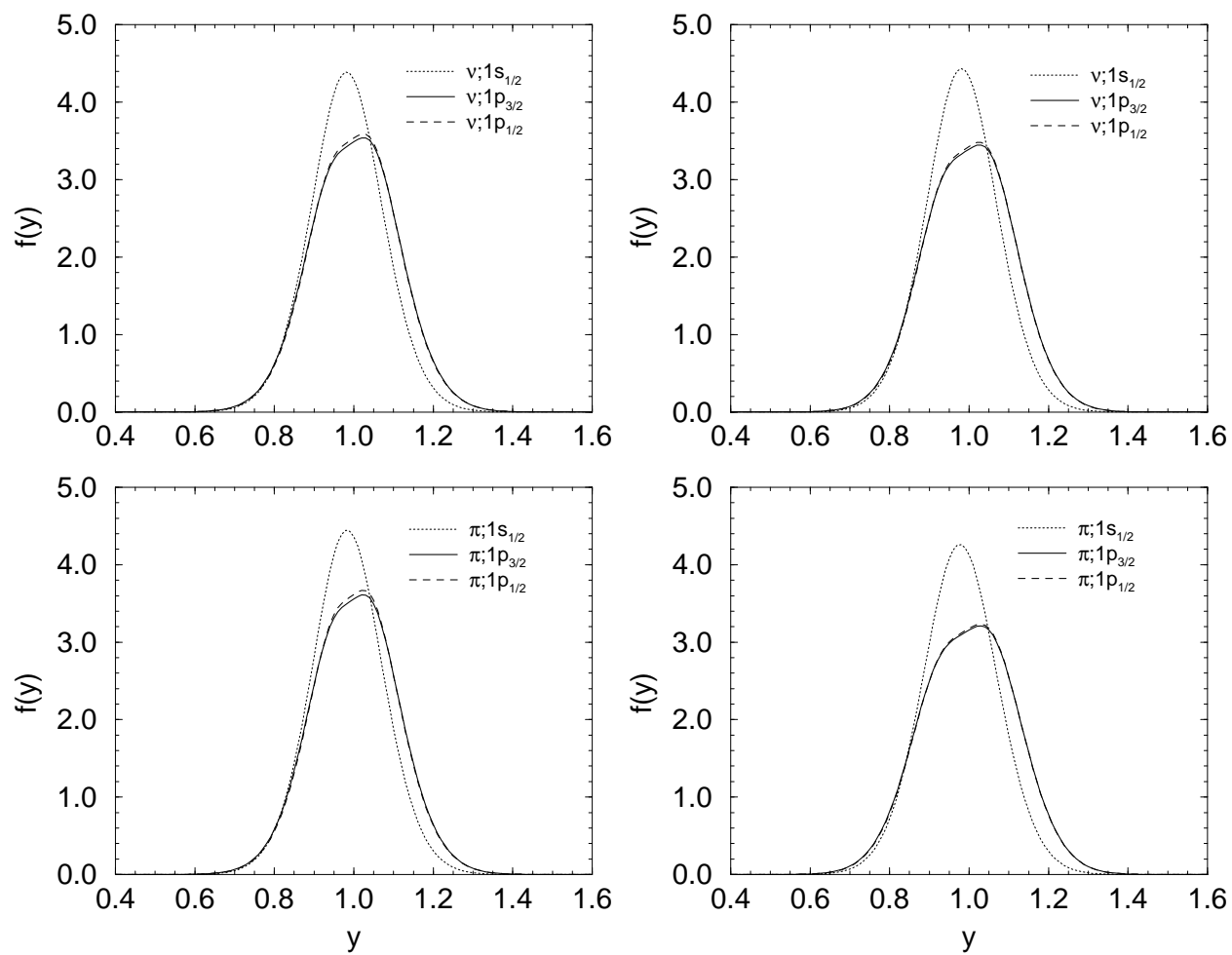

FIG. 5. $f(y)$ for ${ }^{6} \mathrm{Li}$ (left panels) and ${ }^{7} \mathrm{Li}$ (right panels). In each column the top and bottom panels show those of neutrons and protons, respectively. In each panel the dotted, solid and dashed lines denote the distributions of the nucleons in $1 s_{1 / 2}, 1 p_{3 / 2}$ and $1 p_{1 / 2}$ shell orbits, respectively.


FIG. 6. Same as Fig. 5 but for ${ }^{8} \mathrm{Li}$ (left panels) and ${ }^{9} \mathrm{Li}$ (right panels). 

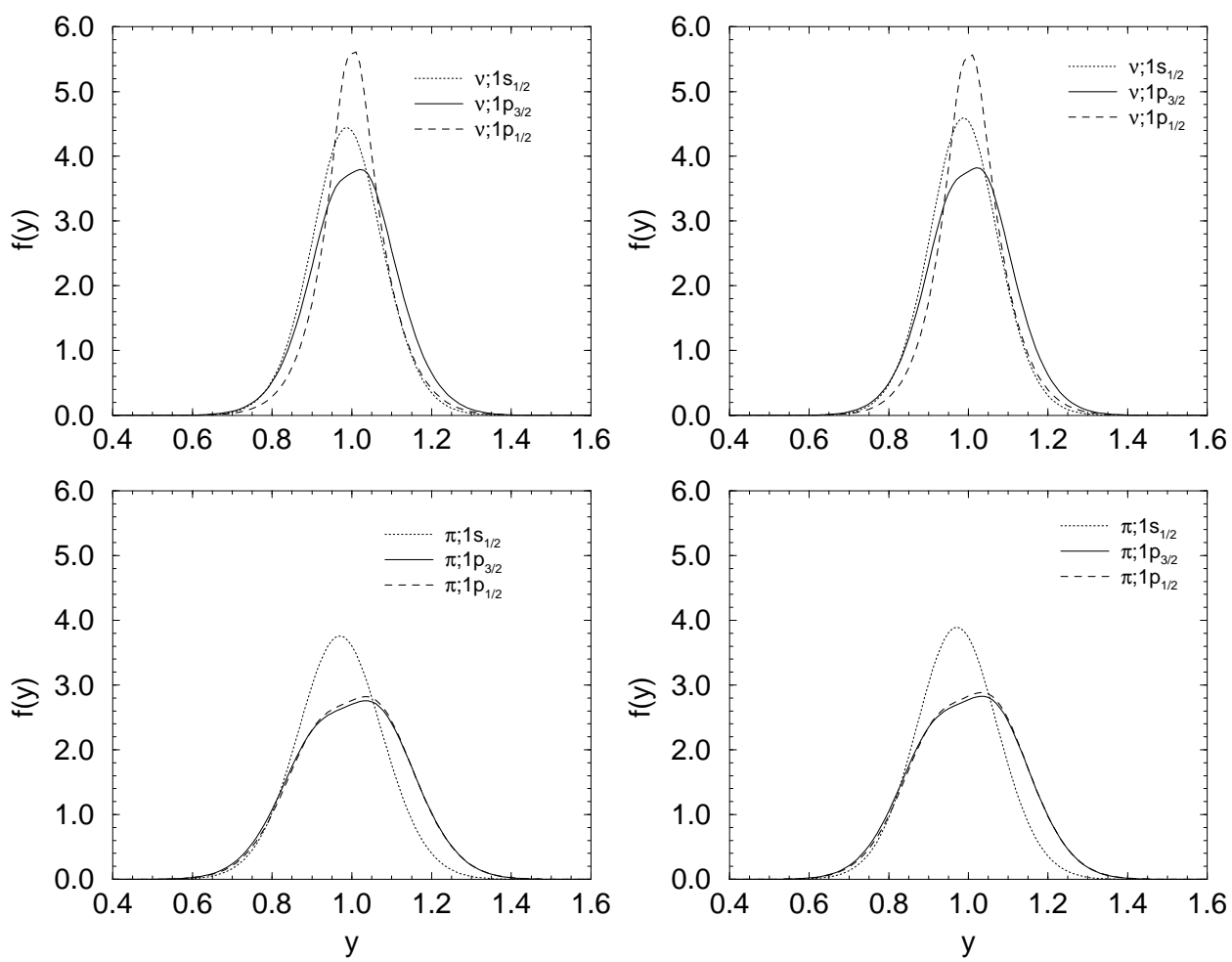

FIG. 7. Same as Fig. 5 but for ${ }^{11} \mathrm{Li}$. The results shown in the left and right panels are for the cases of $S_{n}=301 \mathrm{keV}$ and $350 \mathrm{keV}$, respectively. 


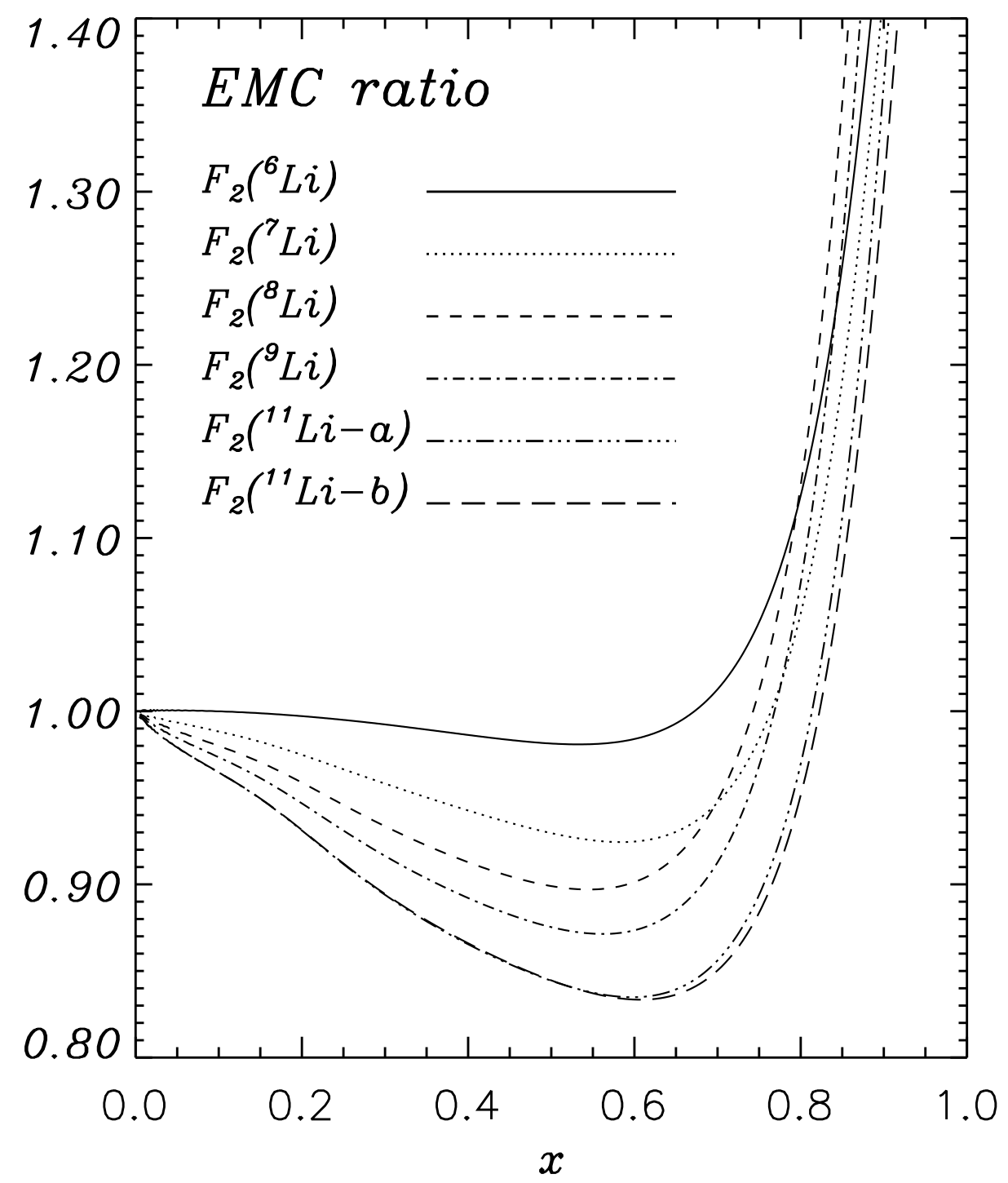

FIG. 8. Ratios of the structure functions of Li isotopes to that of the deuteron at $Q^{2}=10$ $\mathrm{GeV}^{2}$ without isoscalarity corrections. ${ }^{11} \mathrm{Li}-\mathrm{a}(\mathrm{b})$ corresponds to the case of $S_{n}=301(350) \mathrm{keV}$. 


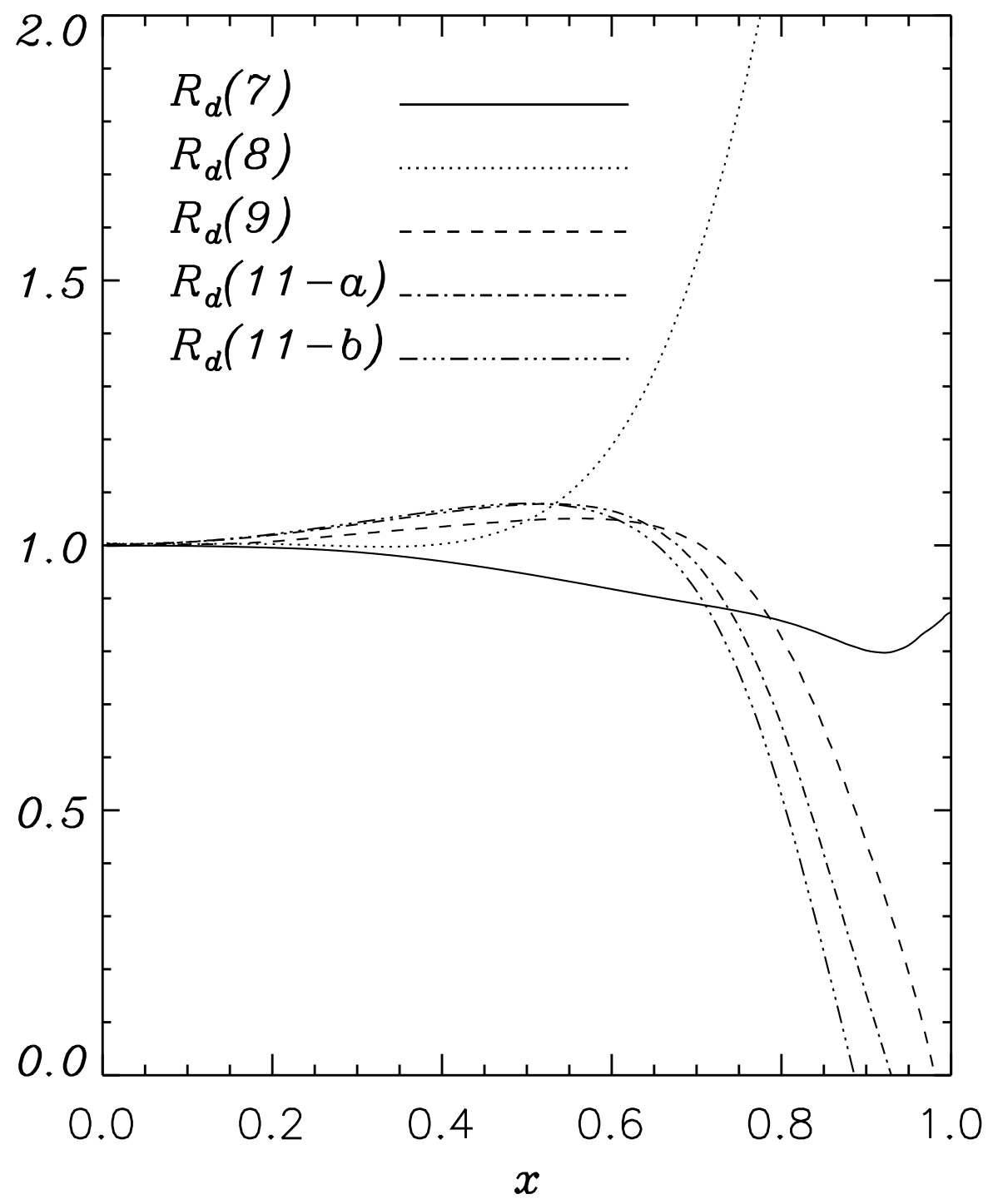

FIG. 9. $R_{d}(A)$ for Li isotopes $\left(Q^{2}=10 \mathrm{GeV}^{2}\right) . \quad R_{d}(11-a(b))$ is for the case of ${ }^{11} \mathrm{Li}$ with $S_{n}=301(350) \mathrm{keV}$. 


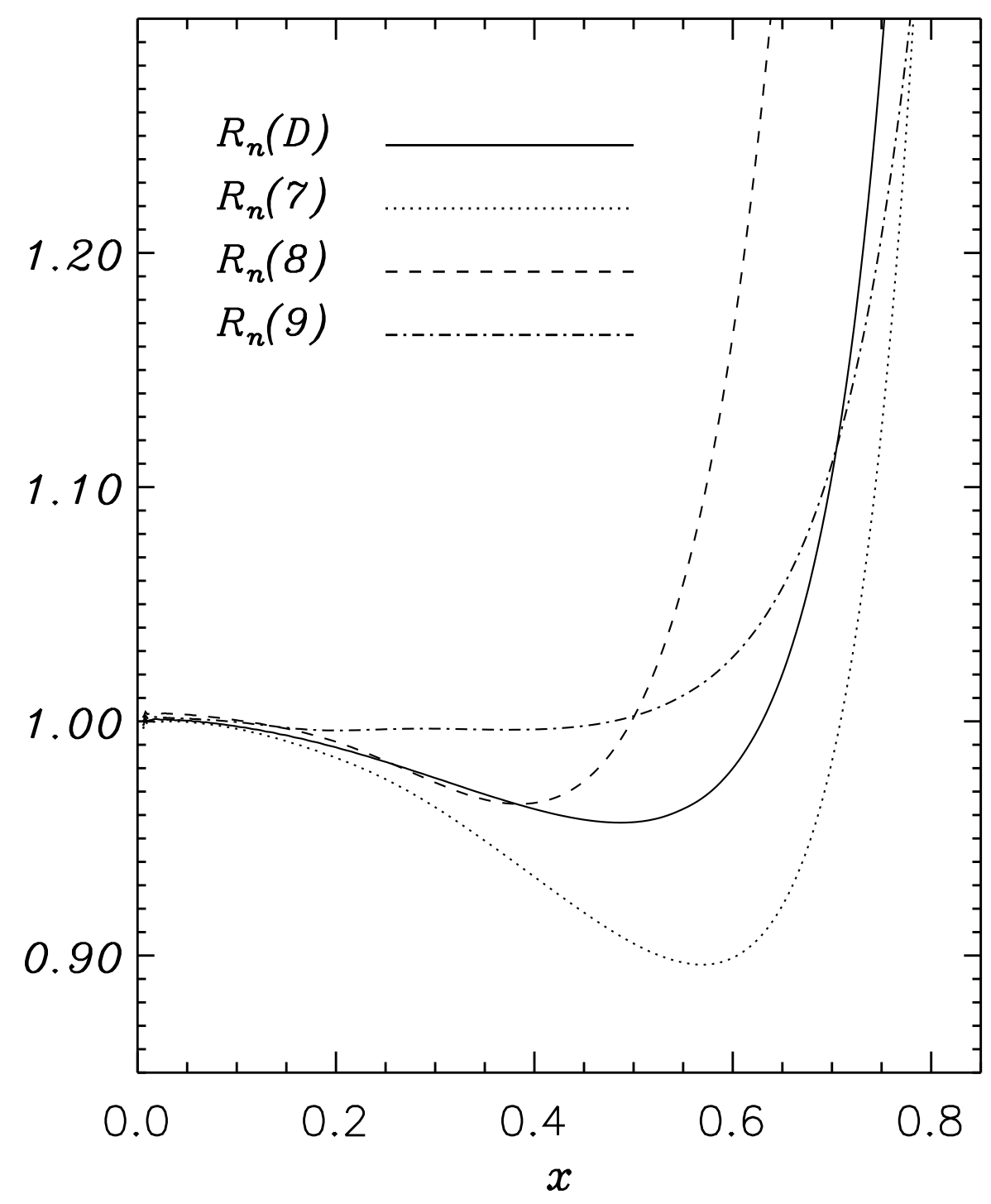

FIG. 10. $R_{n}(A)$ for ${ }^{6-9} \mathrm{Li}$ isotopes $\left(Q^{2}=10 \mathrm{GeV}^{2}\right)$. The ratio of the difference between $F_{2}^{d}$ and $F_{2}^{p}$ to $F_{2}^{n}$ is also presented (denoted by $R_{n}(D)$ ). 


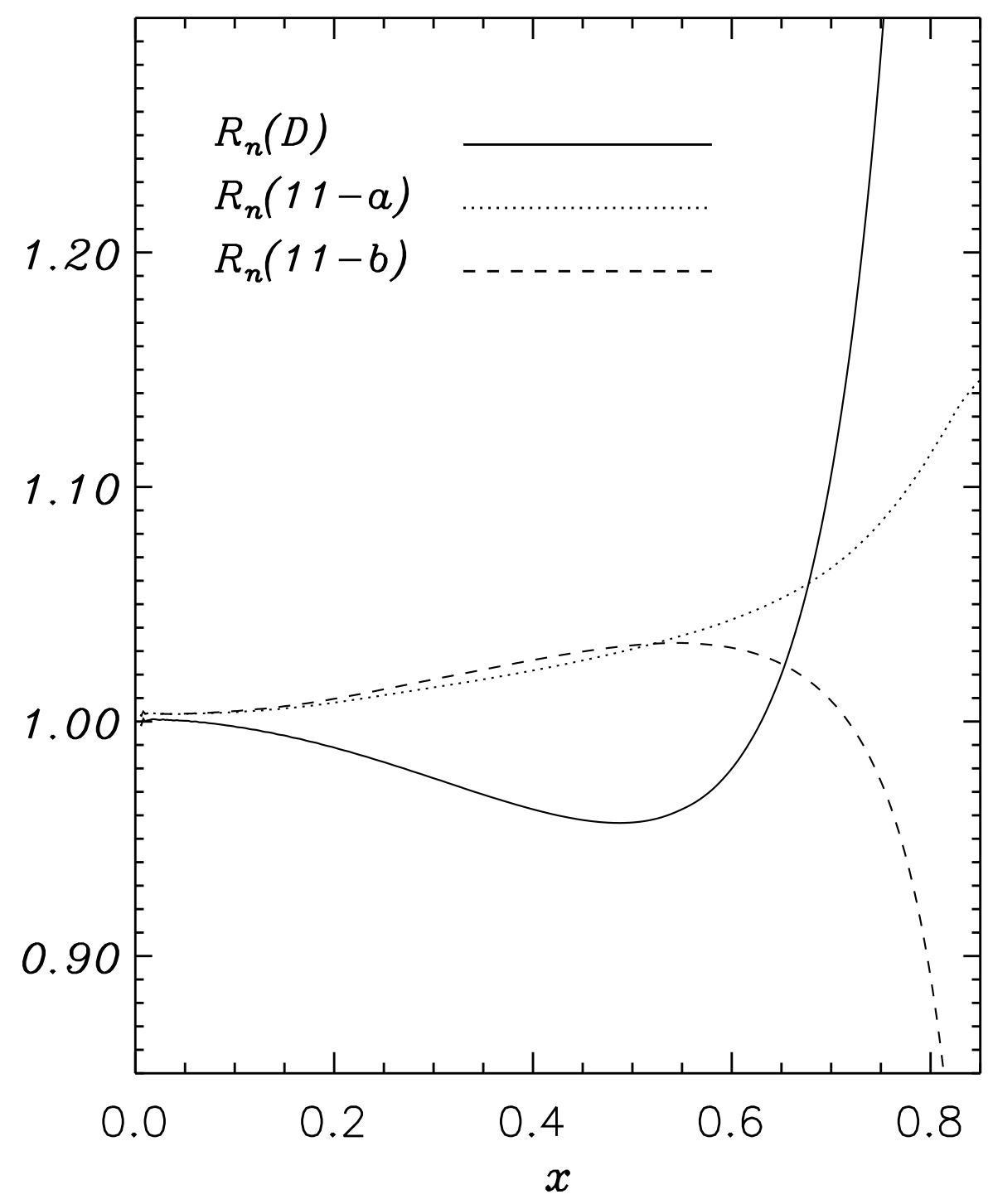

FIG. 11. $R_{n}(A)$ for ${ }^{11} \mathrm{Li}\left(Q^{2}=10 \mathrm{GeV}^{2}\right) . \quad R_{n}(11-a(b))$ is for the case of ${ }^{11} \mathrm{Li}$ with $S_{n}=301(350) \mathrm{keV}$. The ratio $R_{n}(D)$ is also presented for comparison. 


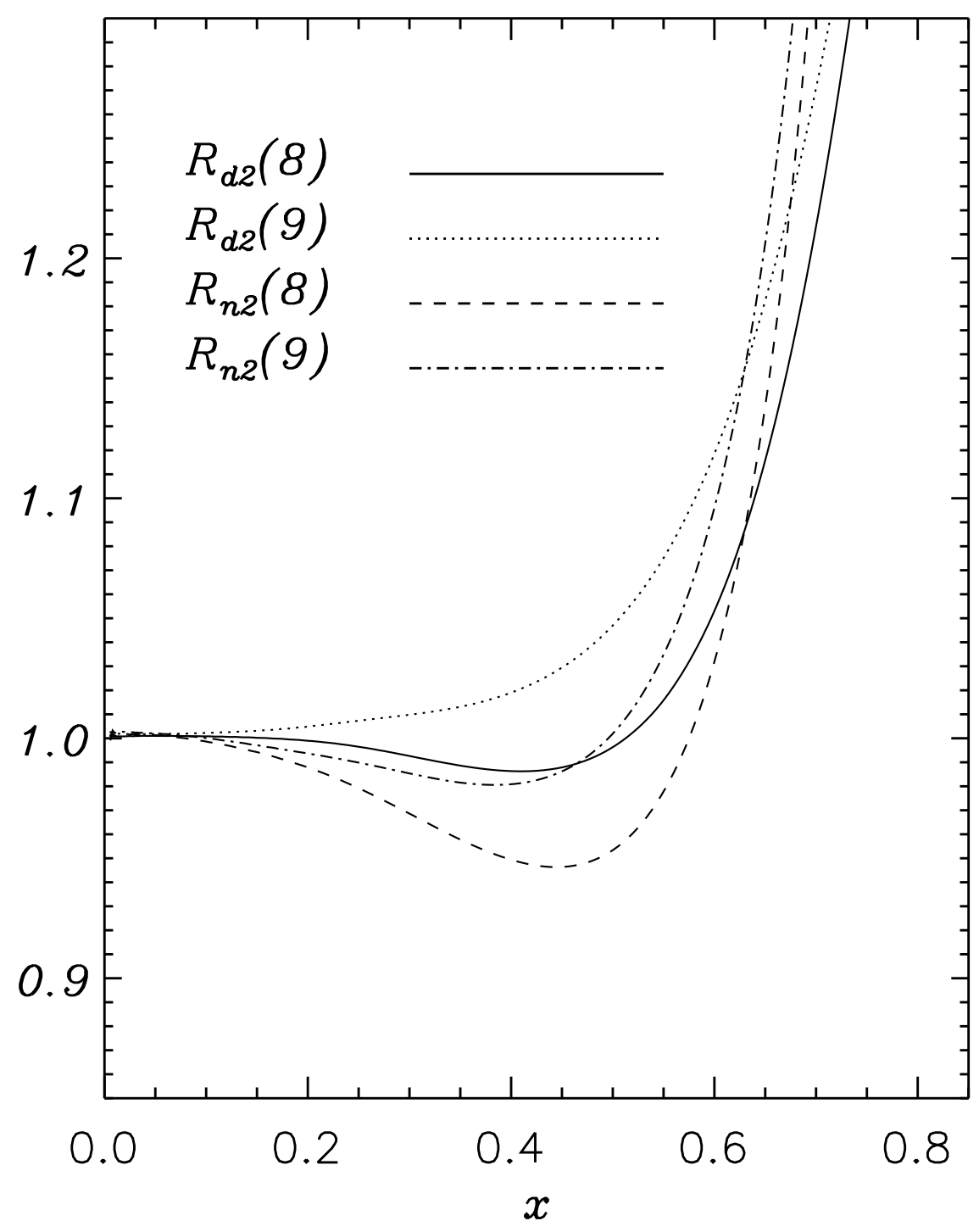

FIG. 12. $R_{d 2}(A)$ and $R_{n 2}(A)$ for ${ }^{8,9} \mathrm{Li}\left(Q^{2}=10 \mathrm{GeV}^{2}\right)$. 

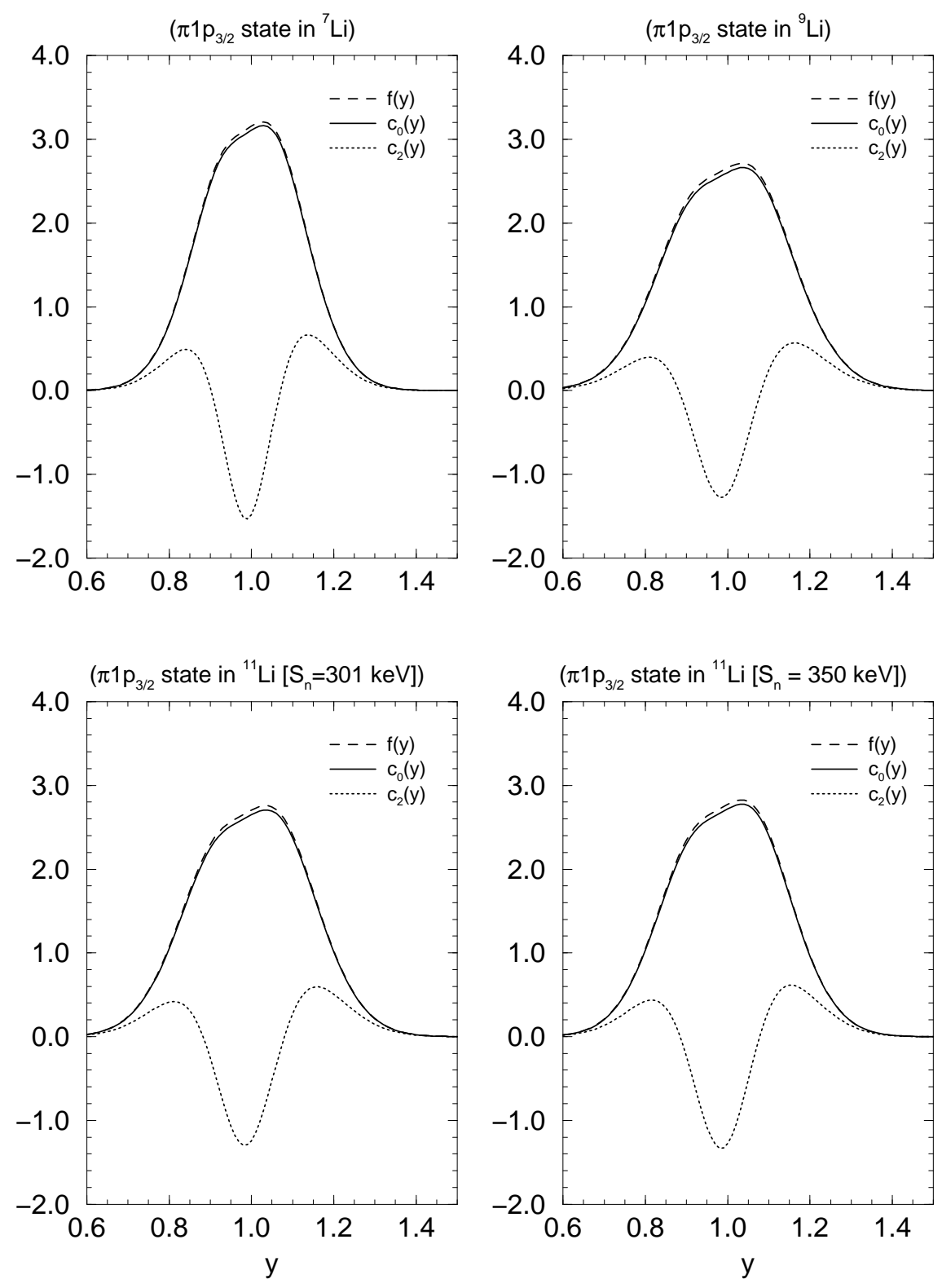

FIG. 13. Distributions, $c_{0}(y)$ and $c_{2}(y)$, for ${ }^{7,9,11} \mathrm{Li}$. For comparison, $f(y)$ is also presented. 


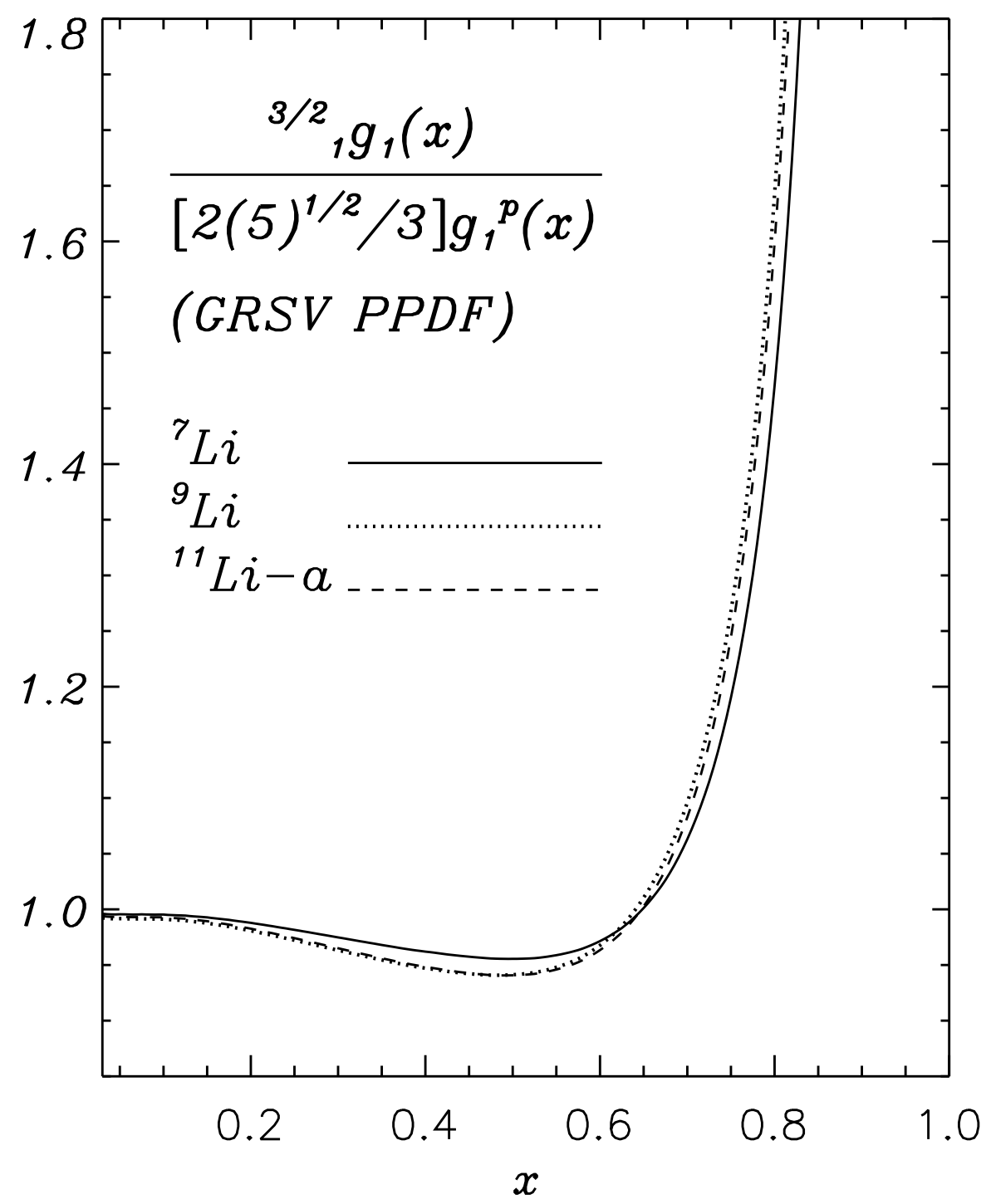

FIG. 14. Ratio of the multipole spin structure function, ${ }_{1}^{3 / 2} g_{1}$, to the usual proton spin structure function, $g_{1}^{p}$. The geometrical factor of $2 \sqrt{5} / 3$ is included in the denominator of the ratio. 


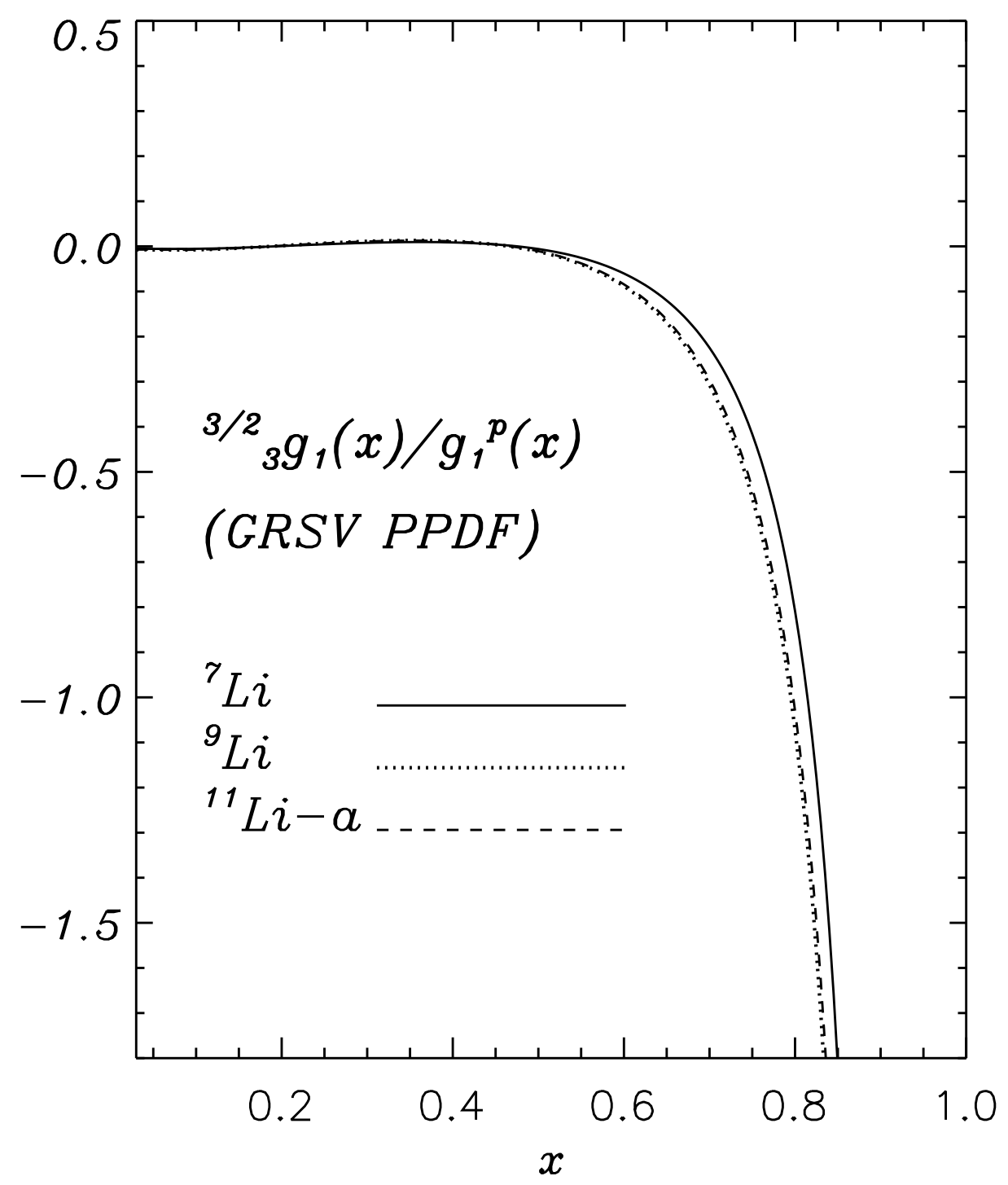

FIG. 15. Ratio of ${ }_{3}^{3 / 2} g_{1}$ to $g_{1}^{p}$. 